\title{
Distribution, evolution and expression of GATA-TFs provide new insights into their functions in light response and fruiting body development of Tolypocladium guangdongense
}

\author{
Chenghua Zhang ${ }^{1}$, Gangzheng Wang ${ }^{1}$, Wangqiu Deng ${ }^{\text {Corresp., } 1}$, Taihui Li ${ }^{\text {Corresp. } 1}$ \\ ${ }^{1}$ State Key Laboratory of Applied Microbiology Southern China, Guangdong Provincial Key Laboratory of Microbial Culture Collection and Application, \\ Guangdong Institute of Microbiology, Guangdong Academy of Sciences, Guangzhou, China \\ Corresponding Authors: Wangqiu Deng, Taihui Li \\ Email address: dengwq@gdim.cn, mycolab@263.net
}

Background. Fungal GATA-type transcription factors (GATA-TFs) are a class of transcriptional regulators involved in various biological processes. However, their functions are rarely analyzed systematically, especially in edible or medicinal fungi, such as Tolypocladium guangdongense, which has various medicinal and food safety properties with a broad range of potential applications in healthcare products and the pharmaceutical industry. Methods. GATA-TFs in T. guangdongense (TgGATAs) were identified using InterProScan. The type, distribution, and gene structure of TgGATAs were analyzed by genome-wide analyses. A phylogenetic tree was constructed to analyze their evolutionary relationships using the neighbor-joining (NJ) method. To explore the functions of GATA-TFs, conserved domains were analyzed using MEME, and cis-elements were predicted using the PlantCARE database. In addition, the expression patterns of TgGATAs under different light conditions and developmental stages were studied using qPCR. Results. Seven TgGATAs were identified. They were randomly distributed on four chromosomes and contained one to four exons. Phylogenetic analysis indicated that GATA-TFs in each subgroup are highly conserved, especially for GATA1 to GATA5. Intron distribution analyses suggested that GATA1 and GATA3 possessed the most conserved gene structures. Light treatments induced the expression levels of TgGATAland TgGATA5-7, but the expression levels varied depending on the duration of illumination. The predicted protein structures indicate that TgGATA1 and TgGATA2 possess typical light-responsive domains and may function as photoreceptors to regulate downstream biological processes. TgGATA3 and TgGATA5 may be involved in nitrogen metabolism and siderophore biosynthesis, respectively. TgGATA6 and TgGATA7 possess unique $\mathrm{Zn}$ finger loop sequences, suggesting that they may have special functions. Furthermore, gene expression analysis indicated that TgGATA1 (WC1) was notably involved in mycelial color transformation, while other genes were involved in fruiting body development to some extent. These results provide valuable information to 
further explore the mechanisms through which TgGATAs are regulated during fruiting body development. 
1 Distribution, evolution and expression of $G A T A-T F s$ provide new insights into their

2 functions in light response and fruiting body development of Tolypocladium guangdongense

3 Chenghua Zhang ${ }^{1}$, Gangzheng Wang1, Wangqiu Deng ${ }^{1 *}$, and Taihui Li ${ }^{1 *}$

4

5 State Key Laboratory of Applied Microbiology Southern China, Guangdong Provincial Key

6 Laboratory of Microbial Culture Collection and Application, Guangdong Institute of

7 Microbiology, Guangdong Academy of Sciences, Guangzhou, 510070, China;

9 Corresponding Author:

10 Wangqiu Deng ${ }^{1}$

11 Guangdong Institute of Microbiology, Building 59, No.100 Courtyard, Xianlie Zhong Road,

12 Yuexiu District, Guangzhou City, Guangdong, 510070, P.R. China,

13 Taihui $\mathrm{Li}^{1}$

14 Guangdong Institute of Microbiology, Building 59, No.100 Courtyard, Xianlie Zhong Road,

15 Yuexiu District, Guangzhou City, Guangdong, 510070, P.R. China,

16 Email address: lith@gdim.cn (T.L.); dengwq@gdim.cn (W.D.); Tel.: +86-020-87137620 (W.D.)

18 Abstract

19 Background. Fungal GATA-type transcription factors (GATA-TFs) are a class of transcriptional 20 regulators involved in various biological processes. However, their functions are rarely analyzed 21 systematically, especially in edible or medicinal fungi, such as Tolypocladium guangdongense, 22 which has various medicinal and food safety properties with a broad range of potential applications 23 in healthcare products and the pharmaceutical industry. 
24 Methods. GATA-TFs in T. guangdongense (TgGATAs) were identified using InterProScan. The 25 type, distribution, and gene structures of TgGATAs were analyzed by genome-wide analyses. A 26 phylogenetic tree was constructed to analyze their evolutionary relationships using the neighbor27 joining (NJ) method. To explore the functions of GATA-TFs, conserved domains were analyzed using MEME, and cis-elements were predicted using the PlantCARE database. In addition, the expression patterns of TgGATAs under different light conditions and developmental stages were studied using qPCR.

Results. Seven $\operatorname{TgGATAs}$ were identified. They were randomly distributed on four chromosomes and contained one to four exons. Phylogenetic analysis indicated that GATA-TFs in each subgroup are highly conserved, especially for GATA1 to GATA5. Intron distribution analyses suggested that GATA1 and GATA3 possessed the most conserved gene structures. Light treatments induced

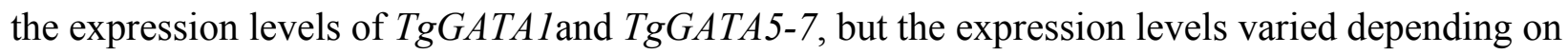
the duration of illumination. The predicted protein structures indicate that TgGATA1 and TgGATA2 possess typical light-responsive domains and may function as photoreceptors to regulate downstream biological processes. TgGATA3 and TgGATA5 may be involved in nitrogen metabolism and siderophore biosynthesis, respectively. TgGATA6 and TgGATA7 possess unique $\mathrm{Zn}$ finger loop sequences suggesting that they may have special functions. Furthermore, gene expression analysis indicated that $\operatorname{Tg} G A T A 1$ (WC1) was notably involved in mycelial color transformation, while other genes were involved in fruiting body development to some extent.

43 These results provide valuable information to further explore the mechanisms through which 44 TgGATAs are regulated during fruiting body development.

\section{Introduction}



fungi, animals, and plants (Patient and McGhee, 2002). Fungal GATA-TFs encode a type IV zincfinger protein and contain one or two zinc finger domains $\left(\mathrm{CX}_{2} \mathrm{CX}_{17-20} \mathrm{CX}_{2} \mathrm{C}\right)$, which bind to the DNA sequence (A/T)GATA(A/G) (Lowry and Atchley, 2000; Park et al., 2006; Chi et al., 2013).

According to the sequence of $\mathrm{CX}_{2} \mathrm{CX}_{17-20} \mathrm{CX}_{2} \mathrm{C}$ domain, fungal GATA-TFs can be grouped into two classes. The first class is the "animal-like" GATA-TFs, which has a leucine in the seventh position of the $\mathrm{Zn}$ finger loop, allowing for hydrophobic contact with the first base of (A/T)GATA(A/G) (Scazzocchio, 2000). In some fungi, the "animal-like" GATA-TFs contain two GATA-type DNA-binding domains, also called 'vertebrate-like' GATA-TFs, wherein the carboxy-terminal domain normally acts as the DNA-binding finger (An et al., 1997; Patient and McGhee, 2002; Lowry and Atchley, 2000). The second category is the 'plant-like' GATA-TFs, which has a glutamic acid residue at position seven of the Zn finger loop (Ballario et al., 1996; Linden and Macino, 1997). This category of GATA-TF generally has a typical PAS domain correlating with light response (Ballario et al., 1996). In addition, in some fungi, there are several other types of GATA-TFs with discrepant sequence features. For instance, in the genome of Saccharomyces cerevisiae, ASH1 is an aberrant GATA-TF with a cysteine residue at the seventh position of the Zn finger loop (Sil and Herskowitz, 1996).

Although the functions performed by GATA-TFs in fungi are very diverse, they generally act as activators or inhibitors to regulate the transcription of a variety of downstream genes, including genes that regulate metabolic processes, cell differentiation and development. These processes are mainly involved in light induction, siderophore biosynthesis, nitrogen metabolism, and matingtype switching (Scazzocchio, 2000; Wong et al., 2008; Hunter et al., 2014; Niehaus et al., 2017).

The 'plant-like' GATA-TFs, such as the photoreceptor proteins white collar-1 (WC-1) and white 
69 collar-2 (WC-2), are induced by light regulating asexual and sexual development, conidiation,

70 fruiting body development, phototropism, resetting of the circadian rhythm, mycelial carotenoid

71 and sterigmatocystin biosynthesis (Rodriguez-Romero, 2010). Ltf1, another 'plant-like' member

72 of GATA-TF in Botrytis cinerea (BcLtf1), is also a photosensitive protein, and regulates the light-

73 dependent differentiation, oxidative stress, and the secondary metabolism (Schumacher et al.,

74 2014). NsdD in Aspergillus nidulans, the homologous protein of BcLtf1, is an activator of sexual

75 development and a key repressor of conidiation (Han et al., 2001; Lee et al., 2014). The 'animal-

76 like' GATA-TFs AreA and AreB in Fusarium fujikuroi participate in the regulation of nitrogen

77 metabolism (Mihlan et al., 2003; Michielse et al., 2014; Pfannmüller et al., 2017). ASD4, another

78 'animal-like' GATA-TF, is a major transcription regulator in the specification of the lineage of 79 asci and ascospores during sexual development in Neurospora crassa (Feng et al., 2000). Urbs-1

80 in the basidiomycete Ustilago maydis and SREA in the Ascomycete A. nidulans, are both

81 'vertebrate-like' GATA-TFs that act as inhibitors, repressing siderophore biosynthesis (An et al.,

82 1997; Lee et al., 2013). In addition, a newly found GATA-TF Ssams2 in Sclerotinia sclerotiorum,

83 which has a threonine residue in the seventh position of the $\mathrm{Zn}$ finger loop, is involved in

84 appressoria formation and chromosome segregation (Liu et al., 2018). However, among the

85 GATA-TFs mentioned above, the light-responsive $\mathrm{WC}-1$ and $\mathrm{WC}-2$, and the nitrogen regulators

86 AreA and AreB, play global roles in fungal growth and development across different species

87 (Niehaus et al., 2017; Pfannmüller et al., 2017). Other GATA-TFs show variable functions in

88 different species, as well as in different stages of development.

89 So far, there have been many reports on the number and functions of GATA-TFs in plants

90 and animals, but only a few studies have systematically analyzed the functions of GATA-TFs in

91 fungi. In lower eukaryotes such as $S$. cerevisiae, the family of GATA-TFs contains over 10 
92 members, and the functions of some members are well known (Lowry and Atchley, 2000;

93 Ronsmans et al., 2019). In some plant pathogenic fungi, such as B. cinerea (Schumacher et al.,

94 2014), F. fujikuroi (Niehaus et al., 2017), S. sclerotiorum (Liu et al., 2018; Li et al., 2018), and

95 Magnaporthe oryzae (Quispe, 2011), several GATA-TFs have been successively identified.

96 Several orthologous proteins have also been identified in A. nidulans (Han et al., 2001; Lee et al.,

97 2013; 2014). In macrofungi, research on GATA-TFs in N. crassa is relatively extensive, with six

98 GATA-TFs being identified and characterized (Feng et al., 2000; Chen et al., 2009); whereas in

99 some edible or medicinal fungi, the functions of 'plant-like' GATA-TFs have been scarcely

100 reported, including the $\mathrm{WC}-1$ and $\mathrm{WC}-2$ in Cordyceps militaris (Yang et al., 2014),

101 Ophiocordyceps sinensis (Yang et al., 2013), Tuber borchii (Ambra et al., 2004), and Lentinula

102 edodes (Sano et al., 2009). In general, the identification and functional analyses of GATA-TFs in

103 edible or medicinal fungi are scattered or insufficiently studied.

104 Light is an important environmental signal for sexual and asexual growth, circadian rhythm 105 and metabolism in fungi (Kamada et al., 2010; Schumacher, 2017). It is known that two GATA-

106 TFs, WC-1 and WC-2, are directly regulated by light, mediating the induction and repression of 107 light-induced genes (Schumacher, 2012; Canessa et al., 2013). WC-1 interacts with WC-2 to form 108 the White Collar Complex (WCC) so as to activate the transcription of downstream light-regulated 109 genes (Corrochano, 2007; Sano et al., 2009; Sanz et al., 2009); however, their functions in 110 different fungi vary. In most fungi, the WCC is involved in asexual conidiospores production and 111 several metabolic pathways (Rodriguez-Romero et al., 2010), whereas in the human pathogenic

112 fungus Cryptococcus neoformans, the WCC is associated with pathogenicity (Idnurm and 113 Heitman, 2005). In several macrofungi, the WCC is involved in fruiting body development and 114 pigment production (Rodriguez-Romero et al., 2010; Yang et al., 2016). Besides, in the edible and 
115 medicinal fungus, $C$. militaris, the production of pharmacologically active ingredients, such as 116 cordycepin, is also regulated by WC-1 (Yang et al., 2016). Therefore, although WC-1 and WC-2

117 are identified as photoreceptors in various fungi, it remains unclear how these proteins regulate the 118 developmental and metabolic processes. species of the genus Tolypocladium in the Ophiocordycipitaceae family described in the Index Fungorum (http://www.indexfungorum.org/). Similar to Ophiocordyceps sinensis, this fungus has various medicinal properties, and its fruiting bodies are safe and non-toxic (Zhang et al., 2018;

123 Zhang et al., 2019). Now it has been considered to be an edible-medicinal fungus, with a broad range of potential applications in health-care products and the pharmaceutical industry. Hence, the mechanism of fruiting body development and biological activities of this species have attracted widespread attention. It is known that GATA transcription factors play vital roles in fungal growth and metabolite synthesis. However, there are very few reports on their characteristics and functions

in T. guangdongense. In this study, the essential features of GATA-TFs in T. guangdongense (TgGATAs) were identified and characterized, including the gene structures, amino acid sequences, phylogenetic relationships, and intron distribution. Furthermore, based on the analyses of conserved motifs, promoters, and gene expression under different light conditions and fruiting

132 body developmental stages, we investigated the functions of TgGATAs.

\section{Materials \& Methods}

\section{Identification of $\operatorname{Tg} G A T A s$}

To identify the TgGATAs, the online analysis platform, InterProScan (Zdobnov and Apweiler, 136 2001) was used to screen the $T$. guangdongense protein database for proteins with conserved 
137 GATA-domains (Zhang et al., 2018). Each GATA-TF candidate sequence was further confirmed

138 by domain analyses using the Pfam protein family database (http://pfam.xfam.org) (Finn et al., 139 2014) and SMART databases (http://smart.emblheidelberg.de/) (Letunic et al., 2012).

\section{Sequence analysis of $\boldsymbol{T g} \boldsymbol{G A T A s}$}

141 The characteristics of the GATA-domains in TgGATAs were analyzed using the ClustalW 142 and BioEdit program (Thompson et al., 1997; Hall, 1999). The DNA and CDS sequences of the 143 predicted GATA-TFs were obtained from the $T$. guangdongense genome. Exon/intron structures 144 were obtained by comparing the cDNA sequences obtained in the previous study (Zhang et al., 145 2019) and the corresponding genomic DNA sequences (Zhang et al., 2018). The isoelectric point 146 (pI) and molecular weight $(\mathrm{Mw})$ of the TgGATAs were calculated using ExPASy tool 147 (http://web.expasy.org/compute pi/). The subcellular localization of each protein was analyzed 148 using BaCelLo Prediction (http:/gpcr.biocomp.unibo.it/bacello/pred.htm). The location of the 149 TgGATAs on the chromosomes was determined using the $T$. guangdongense genome database.

\section{Homologous protein identification and phylogenetic analysis of the GATA family}

151 The homologous proteins of GATAs were identified by genome-wide BLAST analyses.

152 Multiple alignments of GATA-TF protein sequences were performed using the ClustalW program 153 (Thompson et al., 1997). Phylogenetic trees were constructed to analyze the sequence similarity of 154 the GATA factors using the neighbor-joining (NJ) method with a Kimura 2-parameter model in 155 MEGA 5.0 (Tamura et al., 2011). The stability of the internal nodes was assessed by bootstrap 156 analysis of 1,000 replicates. The phylogenetic tree was visualized using iTOL 157 (http://itol.embl.de/help.cgi). Homologous proteins were identified by conducting a BLASTP 
158 search (McGinnis et al., 2004) against forty fungal genomes, which were obtained from the 159 GenBank database or Ensembl Fungi database.

\section{Gene structures and intron analysis of $\boldsymbol{T g} \boldsymbol{G A T A s}$}

161 The online gene structure display server (GSDS) (http://gsds.cbi.pku.edu.cn/) was used to 162 generate the exon-intron structures of the GATA-TFs, including the exon positions and gene 163 lengths (Hu et al., 2015). The position of the introns was determined based on the genome 164 sequences of the selected species.

\section{Conserved motif analysis of $\operatorname{Tg} \boldsymbol{G A T A s}$}

The conserved motifs of the GATA-TFs were studied using the online MEME program 167 (http://meme-suite.org/). Analyses were performed using the following set of parameters: the maximun motif width was set to 50 and the maximum number of motifs was set to 10 . Only the motifs with $\mathrm{P}$ values $<10-6$ and not overlapping with each other were reported. The secondary structures of the GATA-TFs were identified using the SMART databases.

\section{Cis-elements analysis of $\boldsymbol{T g} \boldsymbol{G} \boldsymbol{A} \boldsymbol{T A s}$} were derived from the transcription start site based on the $T$. guangdongense genome (Zhang et al., 2018). The conserved cis-acting regulatory elements present in the promoter regions of the 
For the studies on relative gene expression in response to light, the Tolypocladium

181

182 183

184

185

186

187

188

189

190

191

192

193

194

195

196

197

198

199

200

201

202

guangdongense strain GDGM30035 was cultured on PDA medium at $22 \pm 1^{\circ} \mathrm{C}$ under dark conditions. After 20 days (the colony diameter was approximately half of the diameter of the 90 cm culture dish), samples were exposed to light (1600 lux) for $15 \mathrm{~min}, 30 \mathrm{~min}, 2 \mathrm{~h}$, and $4 \mathrm{~h}$, respectively. Strains cultured under dark conditions were used as controls. Samples were immediately collected at the indicated time points. For the studies on relative gene expression during fruiting body development, the strain was inoculated on liquid YMPD medium (per liter: 3 $\mathrm{g}$ of yeast extract, $10 \mathrm{~g}$ of glucose, $2 \mathrm{~g}$ of malt extract, and $5 \mathrm{~g}$ of peptone) and cultured at $22 \pm 1^{\circ} \mathrm{C}$ with shaking under dark conditions. After 10 days, mycelia were inoculated on rice media (per tissue culture vessels: $25 \mathrm{~g}$ rice, and $20 \mathrm{ml}$ nutrient solution). The composition of the nutrient solution was as follows: $20 \mathrm{~g}$ of sucrose, $5 \mathrm{~g}$ of beef extract, $10 \mathrm{~g}$ of $\mathrm{KNO}_{3}, 4 \mathrm{~g}$ of soybean, and pH 6.0-6.5 per liter. The samples were cultured under dark conditions for two weeks and then shifted to alternating light and dark conditions (10L/14D) based on a previous study (Lin et al., 2009). Samples for RNA extraction were collected at different developmental stages, including vegetative stage $(\mathrm{M}$, the mycelia color is white), color transition period ( $\mathrm{TC}$, the mycelia color changes from white to yellow), primordium ( $\mathrm{P}$, the surface of the hyphae forms primordium), early stage of fruiting body development (FB1, the length of fruiting body is approximately $1-2 \mathrm{~cm}$ ), middle stage of fruiting body development (FB2, the length of fruiting body is approximately 3-4 $\mathrm{cm}$ ), late stage of fruiting body development (FB3, the length of fruiting body is longer than $5 \mathrm{~cm}$ in size), and mature fruiting body (FM, the length of fruiting body is longer than $5 \mathrm{~cm}$ in size and atrovirens). Total RNA was extracted using Trizol, and $1 \mu \mathrm{g}$ of each RNA sample was used for reverse transcription with the HiScript II Q RT SuperMix (+gDNA wiper) (Vazyme). Real-time PCR was conducted in a CFX384 real-time system (Applied Biosystems) using TakaRa SYBR 
203 Premix ExTaq (TakaRa Biotechnology Co.) with specific primers (Supplementary Table 1), and 204 the following parameters: initial preheating at $95^{\circ} \mathrm{C}$ for $30 \mathrm{~s}$, followed by 39 cycles at $95^{\circ} \mathrm{C}$ for $5 \mathrm{~s}$, 205 and $60^{\circ} \mathrm{C}$ for $30 \mathrm{~s}$. The vacuolar protein sorting (VPS) and histone $\mathrm{H} 4(H 4)$ genes were selected as 206 reference genes for analysis the relative expression levels of $\operatorname{TgGATAs}$ at different developmental 207 stages. Based on the previous transcriptome analyses under different illumination times, eight 208 genes (actin, $\alpha$-tub1, $\alpha$-tub2, $\beta$-tub1, rpb, EF1- $\beta, V P S$, and H4) described by Wang et al. (2020) 209 were selected as candidate genes to analyze the expression stability under different light conditions 210 by two statistical algorithms (geNorm and NormFinder). Gene expression levels were calculated 211 using the $2^{-\Delta \Delta \mathrm{Ct}}$ method (Livak and Schmittgen, 2001).

\section{Results}

\section{Identification and characterization of GATA-TFs}

214 Based on the genome sequence of T. guangdongense, seven predicted genes were identified 215 as candidates, accounting for $1.61 \%$ of the total predicted TFs in the T. guangdongense genome. 216 After further confirmation by domain analysis using the Pfam protein family database and SMART 217 database, the seven identified GATA-TFs were denoted as TgGATA1 to TgGATA7 218 (Supplementary Table 2). All the proteins comprised one Cys4 (C4) Zn finger domain, except for 219 TgGATA5, which had two C4 Zn finger domains (Figure 1). In the comparison of the C4 Zn finger 220 domain sequences in different GATA-TFs, four proteins (TgGATA3, TgGATA4, TgGATA5, and 221 TgGATA7) possessed a well-conserved GATA motif, type IVa (Cys- $\mathrm{X}_{2}-\mathrm{Cys}-\mathrm{X}_{17}-\mathrm{Cys}-\mathrm{X}_{2}$-Cys), 222 while the remaining proteins possessed another well-conserved GATA motif, type IVb (Cys- $\mathrm{X}_{2-}$ 223 Cys- $\left.\mathrm{X}_{18}-\mathrm{Cys}-\mathrm{X}_{2}-\mathrm{Cys}\right)$. Based on the amino acid residues in the seventh position of the $\mathrm{Zn}$ finger 224 loops, TgGATA1, TgGATA2, and TgGATA6 belonged to the 'plant-like' GATA-TFs, while 
225 TgGATA3, TgGATA4, TgGATA5 and TgGATA7 were classified as 'animal-like' GATA-TFs.

226 Furthermore, TgGATA5 could also be categorized as a 'vertebrate-like' GATA-TF due to the 227 presence of two GATA-type DNA-binding domains.

228 Detailed information on GATA-TFs is listed in Table 1, including the gene ID, chromosome 229 location, protein length, molecular weight (Mw), the oretical isoelectric point (pI), and prediction 230 of subcellular location. The seven proteins were mapped to four chromosomes in the $T$. 231 guangdongense genome. $\operatorname{TgGATA1}, \operatorname{TgGATA4}$ and $\operatorname{Tg} G A T A 7$ were present on chromosome 1, and 232 the first two had the same orientation, while the third one had opposite orientations. TgGATA2 and $233 \operatorname{TgGATA6}$ were present on chromosomes 2 and 3, respectively. $\operatorname{TgGATA3}$ and $\operatorname{Tg} G A T A 5$ were 234 present on chromosome 4 with the same orientation.Proteins encoded by the predicted GATA-TFs 235 ranged from 195 to 1034 amino acids in length, with an average size of 588 amino acids. The 236 predicted molecular weights of these proteins ranged from 40.49 to $112.10 \mathrm{kDa}$ (average 63.20 $237 \mathrm{kDa}$ ). All the predicted proteins had an isoelectric point (pI) below 10, except for TgGATA6 that 238 had a $\mathrm{pI}$ of 11.08. Based on the analysis of the predicted subcellular localization, only one protein 239 (TgGATA7) was predicted to be localized to the mitochondria, and the rest were predicted to be 240 localized to the nucleus. Nuclear localization signal sequence analyses showed that three proteins 241 had the bipartite-type nuclear localization sequences, including TgGATA1 242 (RKESRPEFGRAIEKARR), TgGATA3 (RRHRKTSIDERRNRKRP) and TgGATA6 243 (RRDSPSADADASGRSRR).

\section{Distribution and phylogenetic analyses of the GATA-TFs family in fungi}

245 An overview of the identified GATA-TFs in T. guangdongense and related homologous 246 proteins in different classes of Ascomycota and Basidiomycota is shown in Table 2. The 247 homologous proteins of five GATA-TFs in T. guangdongense (TgGATA1- TgGATA5) were 
248 identified by genome-wide analyses. Besides, these proteins were highly conserved in 249 Ascomycota, except for Saccharomyces cerevisiae, suggesting that these homologous proteins in 250 different fungi may be the main functional proteins, and may also have similar functions. 251 Compared to Basidiomycota, GATA1 and GATA4 were relatively conserved, while orthologs of 252 the remaining proteins were randomly distributed across different species. Proteins similar to 253 TgGATA6 and TgGATA7 were found in several species. Other GATA-TFs are also listed in Table 254 2. Due to the lower homology, whether some of them are paralogs of the seven types of GATA255 TFs in some cases needs to be further analyzed.

To analyze the sequence similarity between GATA-TF genes in T. guangdongense and those 257 in other fungi, 143 full-length amino acid sequences in Ascomycota were used to construct an 258 unrooted phylogenetic tree using the neighbor-joining (NJ) method. Among these, 43 sequences 259 were from the Eurotiomycetes class, 29 sequences were from the Leotiomycetes class, and 71 260 sequences were from the Sordariomycetes class. These GATA-TFs proteins were classified into 261 seven distinct subgroups, with support values over $90 \%$. The higher supporting rates of each subgroup implied a relatively higher level of synteny between the same types of GATA-TF proteins across different species (Figure 2). Subgroup I was closely related to subgroup $\mathbb{I}$. 264 However, TgGATA1 (CCG_01872) and TgGATA2 (CCG_04254) shared only a 10\% amino acid identity. Although TgGATA6 and TgGATA7 showed lower degrees of identity with those sequence similar proteins listed in Table 2, these proteins were clustered into two subgroups (VI and VII) with $100 \%$ bootstrap values. All proteins in the GATA-TF subgroups I and II were divided into three branches with bootstrap values within $80 \%-90 \%$, and belonged to the three 269 classes of Ascomycota, respectively. The proteins in the GATA-TF subgroups III, IV, and VII were 270 clearly clustered into three classes of Ascomycota with bootstrap values within $90 \%-100 \%$, while 
271 the proteins in GATA-TF subgroups V and VI were grouped into three classes with bootstrap 272 values of $100 \%$. These results indicate that all the proteins in the same subgroups are highly 273 conserved among different classes, even in Ascomycota.

\section{Gene structures and intron distribution of $T g G A T A s$}

275 In order to gain further insight into the evolutionary relationships of the GATA-TFs in $T$. 276 guangdongense, the exon-intron structure for each member of this family was analyzed. The 277 number of exons in the GATA-TFs ranged from one to four (Figure 3). From the comparison of 278 the seven GATA-TFs, the exon-intron structures of GATA2, GATA3, GATA5 and GATA6 were 279 found to be similar, with three exons each. GATA4 had the largest number of exons with four 280 exons, while GATA7 had only one exon. GATA2 had the largest intron sequence among all the 281 GATA-TFs, and GATA1 had the smallest intron sequence.

To determine the relationship between the GATA-TF genes of $T$. guangdongense and its homologous genes in other fungi, the distribution of GATA-TF introns was investigated (Figure

4). The intron numbers of $\operatorname{Tg} G A T A 1$ and $\operatorname{TgGATA3}$ were consistent with the homologous genes in Sordariomycetes, with an intron length between $53 \mathrm{bp}$ and $101 \mathrm{bp}$. The intron positions of GATA1 and GATA3 were also relatively consistent, except for that of GATA1 in Ophiocordyceps sinensis. The introns of GATA1 and GATA3 in the other fungi classes showed random distributions both in terms of the number of introns and their positions. By comparison, significant differences existed in the intron distributions of GATA2 and GATA4 in the same classes, as well as in the same families. However, the intron number of TgGATA4 was consistent with those in Tolypocladium ophioglossoides and Tolypocladium paradoxum, which belong to the same genus. The intron 
293 sinensis. These results indicate that GATA1 and GATA3 are more conserved than other members

294 of the GATA-TFs in the Sordariomycetes class.

\section{Motif analysis of TgGATAs}

296 In order to investigate the functions of TgGATAs, their conserved motifs in $T$. 297 guangdongense and the known functional GATA-TFs in other fungi were identified using the 298 MEME. Ten motifs were identified with lengths varying from 20 to 50 amino acids. Detailed 299 information of the 10 putative motifs is provided in Supplementary Figure 1. As shown in Figure 300 5, the motif compositions of TgGATA1 and TgGATA2 were similar to those of WC- 1 and WC-2 301 in other fungi, which contained two conserved GATA-type Zn finger motifs, and two conserved 302 PAS motifs. These results indicate that TgGATA1 and TgGATA2 are homologues of WC-1 and 303 WC-2, which function as photoreceptors in response to light. Although the amino acid sequence 304 of TgGATA6 shared a lower degree of identity with the homologues of NsdD, these proteins shared similar conserved motifs. The fact that NsdD and its homologues are regulated by light, 306 and TgGATA6 is also classed as a 'plant-like' GATA-TF, suggesting that TgGATA6 is likely to be a downstream target genes activated in response to light. The same dynamics seemed to be found in TgGATA3 and NIT2, as well as in TgGATA5 and SREA, suggesting that TgGATA3 and TgGATA5 may be involved in nitrogen regulation and siderophore biosynthesis, respectively.

310 Both TgGATA4 and TgGATA7 had similar functional motifs as ASD4 in N. crassa (NcASD4), 311 however, the amino acid sequence of TgGATA4 was much closer to that of NcASD4.

313 structures were analyzed using the SMART program (Figure 6A). Like the other white-collar 314 proteins in $C$. militaris and $O$. sinensis, TgGATA1 contained an N-terminal glutamine-rich region, 315 a LOV domain, two PAS domains, and a Zn finger domain. TgGATA2 contains a PAS domain 
316 and a Zn finger domain. However, unlike WC-1 in N. crasssa, TgGATA1 lacked a C-terminal

317 glutamine-rich region. Except for a GATA-type Zn finger domain, TgGATA3 also contains an 318 unknown functional domain DUF1752, while the other proteins, TgGATA4-TgGATA7, had no 319 obvious domains.

Cis-elements and gene expression analyses of $T g G A T A s$ under light

To further explore the functions of TgGATAs in response to light, the cis-elements in their 322 promoter regions were predicted (Figure 6B). In total, ten types of cis-elements were identified, 323 ranging from two to seven in each gene. Among the ten cis-elements, six were responsible for light responsiveness (Box 4, G-box, Sp1, TCT-motif, TCCC-motif, and GATA-motif), three were involved in stress responsiveness (LTR, TC-rich repeats, and MBS), and one was related to circadian control (circadian). In the three 'plant-like' GATA-TFs, TgGATA1 possessed seven cis327 elements, including four light-responsive cis-elements, two stress-responsive cis-elements, and one circadian control cis-element. It is speculated that the expression of TgGATAl may be associated with these biological processes. TgGATA2 only possessed two cis-elements, and both were involved in light responsiveness, while $\operatorname{TgGATA6}$ possessed five light-responsive ciselements, and one stress-responsive cis-element. In the 'animal-like' GATA-TFs, all were found to possess different numbers of light-response cis-elements, however, whether their expression was regulated by light remains to be proven. In addition, $T g G A T A 5$ also possessed a GATA-motif, 334 suggesting that this gene may interact with other GATA-TFs genes.

To test the above hypotheses, the expression of GATA-TFs in T. guangdongense was further 336 analyzed under different light conditions by quantitative real-time PCR. Based on the results on reference genes, $\alpha$-tubl and $\beta$-tubl were selected as reference genes for analysis of relative expression levels of TgGATAs under different light conditions (Supplementary Table 8). TgGATA1 
339

340

341

342

343

344

345

346

347

348

349

350

351

352

353

354

355

356

357

358

359

360

and $\operatorname{TgGATA} 2$ exhibited slight up-regulation when $\beta$-tubl was used as the reference gene (Figure 7B), however, no difference was observed when $\alpha-t u b 1$ was used as the reference gene (Figure 7A). After light treatment for $30 \mathrm{~min}$, the relative expression level of $T g G A T A 1$ decreased, while the relative expression level of $\operatorname{TgGATA2}$ showed a slight up-regulation with no significant difference. The relative expression levels of $\operatorname{Tg} G A T A 3$ and $\operatorname{TgGATA4}$ were down-regulated, but only the expression of $\operatorname{TgGATA4}$ was significantly decreased after light treatment. The relative expression levels of $\operatorname{TgGATA5}$ and $\operatorname{TgGATA6}$ remained up-regulate patterns after light treatment, and their expression levels changed more markedly after light treatment for $30 \mathrm{~min}$. The relative expression level of $\operatorname{TgGATA7}$ also exhibited an up-regulate pattern, however, a significant difference was observed in light treatment for $4 \mathrm{~h}$.

\section{Expression analysis of $\operatorname{Tg} G A T A s$ during fruiting body development}

To better understand the functions of GATA-TFs in T. guangdongense, the expression patterns of $\operatorname{TgGATAs}$ were studied at the different developmental stages of the fruiting body (Figure 8). During developmental stages, $\operatorname{TgGATA1}$ was significantly up-regulated at the stage where hyphal color changed from white to yellow (TC). TgGATA2 was obviously up-regulated from the primordium formation stage (P) to the fruiting body developmental stages (FB1 and FB2). The expression level of TgGATA3 significantly increased at the mature fruiting body stage (FM). The relative expression levels of $\operatorname{TgGATA4}$ and $\operatorname{TgGATA5}$ exhibited down-regulation trends mainly from the TC stage to FB2 stages, and changed more obviously at the TC stage. TgGATA6 was significantly up-regulated at the late stage of fruiting body development (FB3) and mature fruiting body stage. TgGATA7 maintained continuous up-regulation from the primordium formation stage to mature fruiting body stage. Although the expression levels of all genes changed 
361 in obviously varying degrees at some stages by normalizing with VPS and H4 (Figure 8A and B),

362 the varying trends were consistent.

363 Discussion

364

GATA-TFs are widely distributed in fungi, animals, and plants (Patient and McGhee, 2002).

365

However, the number of GATA-TFs varies greatly within and between the three kingdoms. In

366

plants, GATA-TFs have been systematically characterized in many species, such as Arabidopsis

367

thaliana (Reyes et al., 2004), Gossypium raimondii, G. arboretum, G. hirsutum (Zhang et al.,

368

2019), and Vitis vinifera (Zhang et al., 2018), where the number of GATA-TFs ranges from 19 to

369

87. In vertebrates, six GATA-TFs have been identified with well-characterized functions in disease

370

control (Lentjes et al., 2016; Tremblay et al., 2018), and 11 GATA-TFs have been identified in

371 Caenorhabditis elegans, (Block and Shapira, 2015). Over ten GATA-TFs have been found in yeast

372 (Ronsmans et al., 2019). While in other fungi, 3 to 16 members of GATA-TFs have been found

373 with the help of genome-wide analyses (Park et al., 2006). In previous studies, very few GATA-

374 TFs have been analyzed in some edible or medicinal fungi, and the total number of GATA-TFs

375 and their functions remain little known. The present study is the first to systematically analyze the

376 GATA-TFs in the edible and medicinal fungus, T. guangdongense. As a result, seven GATA-TFs

377 were identified, indicating that fungi might possess relatively fewer GATA-TFs than that in plants.

According to the domain features, two 'plant-like' GATA-TFs, GATA1 (homolog of WC-1)

379

and GATA2 (homolog of WC-2), were classified as photoreceptors, which are widely distributed

380

in Ascomycota (Rodriguez-Romero et al., 2010). However, there is one exception, S. cerevisiae

381 lacks light responses and WC photoreceptors do not exist in this fungus (Park et al., 2006). WC-

3821 and WC-2 have also been found in Zygomycetes and Basidiomycetes (Corrochano and Garre,

383 2010; Rodriguez-Romero et al., 2010). However, a homolog of WC-2 was identified in

Peer) reviewing PDF | (2020:01:44976:2:1:NEW 21 Jul 2020) 
384 Coprinopsis cinerea and Cryptococcus neoformans with a relatively lower identity compared to 385 those in Ascomycota (Park et al., 2006; Corrochano, 2007; Kuratani et al., 2010), indicating that 386 GATA1 is more conserved than GATA2 in fungi. Protein domain and intron distribution analyses 387 also suggested that GATA-TFs in the GATA1 subgroup shared similar gene structure and protein 388 domain. As WC-1 often interacts with WC-2 to form WCC, we further analyzed the expression 389 correlations of TgGATAs based on previous transcriptome data (Zhang et al., 2019) by weighted 390 correlation network analysis (WGCNA) according to the method described by Langfelder and 391 Horvath (2008), following the general WGCNA guidelines (Zhang and Horvath, 2005) 392 (Supplementary Table 9). Co-expression network analysis showed that significant positive 393 regulatory relationships exist between $\operatorname{TgGATA1}$ and $\operatorname{TgGATA2}$, with a pairwise Pearson 394 correlation coefficients of $0.88(\boldsymbol{P}=1.13 \mathrm{E}-08)$. Additionally, the WGCNA analysis results also 395 suggested that the possible regulatory connections may exist between $\operatorname{TgGATA1-TgGATA7}$ and 396 TgGATA2-TgGATA7, with the pairwise Pearson correlation coefficients of $0.75(P=1.82 \mathrm{E}-05)$ and $3970.82(P=9.34 \mathrm{E}-07)$, respectively. These results suggested that $\operatorname{TgGATA7}$ may be a downstream 398 target gene of WCC; however, this hypothesis needs to be confirmed in further studies. The 399 'animal-like' GATA-TFs in fungi contained four members, and all of which are likely to be found 400 in both Ascomycota and Basidiomycota. Based on the conserved domains of homologous proteins, 401 TgGATA3 and TgGATA5 may have functions similar to AreA and SreA, which are found to be 402 mainly involved in the regulation of the nitrogen metabolism and siderophore biosynthesis, 403 respectively. AreA acts as a positive regulator of nitrogen metabolite repression (NMR) sensitive 404 genes involved in the utilization of alternative nitrogen sources, while SreA deficiency not only 405 leads to the repression of siderophore biosynthesis but also results in the deregulation of 406 siderophore-bound iron uptake and ornithine esterase expression (Oberegger et al., 2001). 
407 TgGATA4 is highly homologous with AreB or ASD4. Apart from being involved in the regulation 408 of the nitrogen metabolism (Michielse et al., 2014), AreB also acts as a strong repressor of 409 bikaverin gene expression (Pfannmüller et al., 2017). In N. crassa, ASD4 is involved in ascus and 410 ascospore development (Feng et al., 2000). On the basis of these findings, the association of 411 TgGATA4 with developmental processes or the regulation of metabolic processes needs to be 412 further investigated. regulators AreA/NIT2 and AreB (or the sexual development regulator ASD4), the central 415 components of the blue light-sensing system WC-1 and WC-2, the sexual and asexual development regulator NsdD/SUB-1(Han et al., 2001; Colot et al., 2006; Lee et al., 2014), the iron uptake 417 regulator SreA (Machida and Gomi, 2010), SFH1 (involved in hyphal growth, reactive oxygen 418 species accumulation, and pathogenicity) (Liu et al., 2018a), and AMS2 (associated with 419 appressoria formation and chromosome segregation) (Liu et al., 2018b). In T. guangdongense, 420 homologs of AreA, AreB/ASD4, WC-1, WC-2, and SreA were found, and the alignments of the 421 homologous GATA-domains were very similar with high degrees of identity (Supplementary 422 Figure S2A-E). However, no reliable homologs of NsdD/SUB-1, SFH1 and AMS2 were found in 423 T. guangdongense. Although the phylogenetic analysis of TgGATA6 was clustered into 424 NsdD/SUB-1 with an approval rate of 100\%, the amino acid sequence of TgGATA6 showed 425 significant differences from those of NsdD/SUB-1 and their homologs. This phenomenon was a 426 little strange, and a probable alternative splicing in this gene under different light conditions may 427 be the cause. Phylogeny and domain analyses also indicated that TgGATA7 was significantly 428 different from the other proteins. Furthermore, alignments of the GATA-domains between the 429 above two proteins and the known functional proteins demonstrated that TgGATA6 possesses 
430 similar amino acid sequences of the $\mathrm{Zn}$ finger loop with those in AnNsdD, SsNsd1, BcLtf1,

431 NcSUB1, and Ffcsm1, while TgGATA7 process the unique amino acid sequences of the $\mathrm{Zn}$ finger

432 loop (Supplementary Figure 2F). As a result, TgGATA6 may be classified into subgroups VI, and

433 TgGATA7 may have a new function in T. guangdongense.

Genes that respond to light can be grouped into two classes: early and late light-induced genes. As a first response, light-activated White-Collar Complex (WCC) binds to the promoters of early light-responsive genes, such as frq, vivid, and sub-1, to transiently induce or repress their expression (Yu and Fischer, 2018). In the present study, the expression level of TgGATA1 slightly increased when exposed to light for $15 \mathrm{~min}$, but decreased when exposed to light from $30 \mathrm{~min}$ to

$4 \mathrm{~h}$, suggesting that the expression level of WC1 may peak within the first $30 \mathrm{~min}$. This phenomenon after light treatment for 30min was different from that in Tuber borchii, C. militaris and $O$. sinensis, in which $W C 1$ was significantly up-regulated within $30 \mathrm{~min}$ of light treatment (Ambra et al., 2004; Yang et al., 2013; 2014). Besides, the expression levels of TgGATA5 and

$443 \operatorname{TgGATA6}$ were increased significantly within $30 \mathrm{~min}$ of light treatment, suggesting that they may 444 function as early light-induced genes to regulate the downstream target genes. After the first wave 445 of the gene induction, the WCC and other transcriptional factors activate the expression of the late 446 light-responsive genes to regulate the expression of downstream genes (Yu and Fischer, 2019). In 447 this study, $\operatorname{TgGATA7}$ displayed a significant increasing expression pattern after exposure to light 448 for $4 \mathrm{~h}$, suggesting that this gene may be a late light-induced gene or light-induced downstream 449 target gene. 
452 this study, all the seven GATA-TFs in T. guangdongense showed differential expression patterns 453 during fruiting body development. Our results suggested that TgGATA1 (WC1) was notably 454 involved in the mycelia color shift, while TgGATA2 (WC2) was involved in the fruiting body 455 development. TgGATA3 was up-regulation at the mature fruiting body stage (FM), suggesting that 456 it may be related to metabolic processes, such as pigment metabolism. TgGATA4 and TgGATA5 457 were down-regulated at stages of fruiting body development, suggesting that they may negatively 458 regulate the sexual development. In contrast, $\operatorname{Tg} G A T A 6$ and $\operatorname{Tg} G A T A 7$ were up-regulated at stages 459 of fruiting body development, suggesting that they may positively regulate the sexual 460 development. However, these results are only the preliminary findings with respect to the 461 relationship between $\operatorname{TgGATAs}$ and sexual development. The specific functions of these genes 462 require further investigation through genetic approaches.

\section{Conclusions}

464 In this study, seven GATA-TFs were identified in T. guangdongense. TgGATA1 and 465 TgGATA2 can be considered photoreceptors based on their phylogeny, conserved domains, and 466 expression patterns. It was considered that TgGATA3 and TgGATA5 may be involved in nitrogen 467 metabolism and siderophore biosynthesis, respectively, based on the results of motif and 468 homologous analyses. Three genes (TgGATA5-7) were significantly induced by light, while all 469 TgGATAs were involved in fruiting body development to some extent. However, how these genes 470 regulate the fruiting body development should be further analyzed by gene knockout or other 471 genetic approaches. The present results provide comprehensive information on fungal GATA-TFs 472 and lay the foundation for further functional studies on TgGATAs.

\section{Acknowledgements}


This work was funded by the grants from the National Natural Science Foundation of China

475 (Project No. 31800012) and the Science and Technology Planning Project of Guangzhou (No.

201804020018), the Science and Technology Planning Project of Guangdong Province, China

477 (No. 2018A0303130164), and GDAS' Project of Science and Technology Development (No.

478 2020GDASYL-20200104011).

\section{References}

480

481

482

483

484

485

486

487

488

489

490

491

492

493

494

495

496

497

498

499

500

501

Ambra R, Grimaldi B, Zamboni S, Filetici P. 2004. Photomorphogenesis in the hypogeous fungus Tuber borchii: isolation and characterization of Tbwc-1, the homologue of the bluelight photoreceptor of Neurospora crassa. Fungal Genetics and Biology 41(7): 688-697

An Z, Zhao Q, McEvoy J, Yuan W-M, Markley J-L, Leong S-A. 1997. The second finger of Urbs1 is required for iron-mediated repression of sid1 in Ustilago maydis. Proceedings of the National Academy of Sciences of the United States of America 94(11):5882-5887

Ballario P, Vittorioso P, Magrelli A, Talora C, Cabibbo A, Macino G. 1996. White collar-1, a central regulator of blue light responses in Neurospora, is a zinc finger protein. Embo Journal 15(7): 1650-1657

Block D-H, Shapira M. 2015. GATA transcription factors as tissue-specific master regulators for induced responses. Worm 4: e1118607

Borkovich K-A, Alex L-A, Yarden O, Freitag M, Turner G-E, Read N-D, Seiler S, BellPedersen D, Paietta J, Plesofsky N, Plamann M, Goodrich-Tanrikulu M, Schulte U, Mannhaupt G, Nargang F-E, Radford A, Selitrennikoff C, Galagan J-E, Dunlap J-C, Loros J-J, Catcheside D, Inoue H, Aramayo R, Polymenis M, Selker E-U, Sachs M-S, Marzluf G-A, Paulsen I, Davis R, Ebbole D-J, Zelter A, Kalkman E-R, O'Rourke R, Bowring F, Yeadon J, Ishii C, Suzuki K, Sakai W, Pratt R. 2004. Lessons from the genome sequence of Neurospora crassa: tracing the path from genomic blueprint to multicellular organism. Microbiology and Molecular Biology Reviews 68: 1-108

Canessa P, Schumacher J, Hevia M-A, Tudzynski P, Larrondo L-F. 2013. Assessing the e $\square$ ects of light on di $\square$ erentiation and virulence of the plant pathogen Botrytis cinerea: characterization of the White Collar Complex. PLoS One 8: e84223 
502

503

504

505

506

507

508

509

510

511

512

513

514

515

516

517

518

519

520

521

522

523

524

525

526

527

528

529

530

531

532

Chen C-H, Ringelberg C-S, Gross R-H, Dunlap J-C, Loros J-J. 2009. Genome-wide analysis of light-inducible responses reveals hierarchical light signalling in Neurospora. Embo Journal 28(8): 1029-1042

Chi Z, Wang X-X, Geng Q, Chi Z-M. 2013. Role of a GATA-type transcriptional repressor Sre1 in regulation of siderophore biosynthesis in the marine-derived Aureobasidium pullulans HN6.2. Biometals 26: 955-967

Colot H-V, Park G, Turner G-E, Ringelberg C, Crew C-M, Litvinkova L, Weiss R-L, Borkovich K-A, Dunlap J-C. 2006. A high-throughput gene knockout procedure for Neurospora reveals functions for multiple transcription factors. Proceedings of the National Academy of Sciences of the United States of America 103(27):10352-10357.

Corrochano L-M. 2007. Fungal photoreceptors: sensory molecules for fungal development and behaviour. Photochemical and Photobiological Sciences 6(7): 725-736

Corrochano L-M, Garre V. 2010. Photobiology in the Zygomycota: Multiple photoreceptor genes for complex responses to light. Fungal Genetics and Biology 47(11): 893-899

Dean R-A, Talbot N-J, Ebbole D-J, Farman M-L, Mitchell T-K, Orbach M-J, Thon M, Kulkarni R, Xu J-R, Pan H-Q, Read N-D, Lee Y-H, Carbone I, Brown D, Oh Y-Y, Donofrio N, Jeong J-S, Soanes D-M, Djonovic S, Kolomiets E, Rehmeyer C, Li W-X, Harding M, Kim S, Lebrun M-H, Bohnert H, Coughlan S, Butler J, Calvo S, Ma L-J, Nicol R, Purcell S, Nusbaum C, Galagan J-E, Birren B-W. 2005. The genome sequence of the rice blast fungus Magnaporthe grisea. Nature 434: 980-986

Dujon B, Sherman D, Fischer G, Durrens P, Casaregola S, Lafontaine I, De Montigny J, Marck C, Neuvéglise C, Talla E, Goffard N, Frangeul L, Aigle M, Anthouard V, Babour A, Barbe V, Barnay S, Blanchin S, Beckerich J-M, Beyne E, Bleykasten C, Boisramé A, Boyer J, Cattolico L, Confanioleri F, De Daruvar A, Despons L, Fabre E, Fairhead C, Ferry-Dumazet H, Groppi A, Hantraye F, Hennequin C, Jauniaux N, Joyet P, Kachouri R, Kerrest A, Koszul R, Lemaire M, Lesur I, Ma L, Muller H, Nicaud J-M, Nikolski M, Oztas S, Ozier-Kalogeropoulos O, Pellenz S, Potier S, Richard G-F, Straub M-L, Suleau A, Swennen D, Tekaia F, Wésolowski-Louvel M, Westhof E, Wirth B, Zeniou-Meyer M, Zivanovic I, Bolotin-Fukuhara M, Thierry A, Bouchier C, Caudron B, Scarpelli C, Gaillardin C, Weissenbach J, Wincker P, Souciet J-L. 2004. Genome evolution in yeasts. Nature 430: 35-44 
533 Feng B, Haas H, Marzluf G-A. 2000. ASD4, a new GATA factor of Neurospora crassa, displays

534

535

536

537

538

539

540

541

542

543

544

545

546

547

548

549

550

551

552

553

554

555

556

557

558

559

560

561

562

563 sequence-specific DNA binding and functions in ascus and ascospore development. Biochemistry 39(36): 11065-11073

Finn R-D, Bateman A, Clements J, Coggill P, Eberhardt R-Y, Eddy S-R, Heger A, Hetherington K, Holm L, Mistry J, Sonnhammer E-L-L, Tate J, Punta M. 2014. Pfam: the protein family database. Nucleic Acids Research 42(1): 222-230

Hall T-A. 1999. BioEdit: a user-friendly biological sequence alignment editor and analysis program for Windows 95/98/NT. Nucleic Acids Symposium Series 41:95-98

Han K-H, Han K-Y, Yu J-H, Chae K-S, Jahng K-Y, Han D-M. 2001. The nsdD gene encodes a putative GATA-type transcription factor necessary for sexual development of Aspergillus nidulans. Molecular Microbiology 41: 299-309

Hu B, Jin J, Guo A-Y, He Z, Luo J, Gao G. 2015. GSDS 2.0: an upgraded gene feature visualization server. Bioinformatics 31(8): 1296-1297

Hunter C-C, Siebert K-S, Downes D-J, Wong K-H, Kreutzberger S-D, Fraser J-A, Clarke D-F, Hynes M-J, Davis M-A, Todd R-B. 2014. Multiple nuclear localization signals mediate nuclear localization of the GATA transcription factor AreA. Eukaryotic Cell 13(4): 527-538

Idnurm A, Heitman J. 2005. Light controls growth and development via a conserved pathway in the fungal kingdom. PLoS Biology. 3(4): 615-626

Kamada T, Sano H, Nakazawa T, Nakahori K. 2010. Regulation of fruiting body photomorphogenesis in Coprinopsis cinerea. Fungal Genetics and Biology 47(11): 917-921

Kamper J, Kahmann R, Bolker M, Ma L-J, Brefort T, Saville B-J, Banuett F, Kronstad JW, Gold S-E, Müller O, Perlin M-H, Wösten H-A-B, de Vries R, Ruiz-Herrera J, Reynaga-Peña C-G, Snetselaar K, McCann M, Pérez-Martín J, Feldbrügge M, Basse CW, Steinberg G, Ibeas J-I, Holloman W, Guzman P, Farman M, Stajich J-E, Sentandreu R, González-Prieto J-M, Kennell J-C, Molina L, Schirawski J, Mendoza-Mendoza A, Greilinger D, Münch K, Rössel N, Scherer M, Vraneš M, Ladendorf O, Vincon V, Fuchs U, Sandrock B, Meng S-W, Ho E-C-H, Cahill M-J, Boyce K-J, Klose J, Klosterman S-J, Deelstra H-J, Ortiz-Castellanos L, Li W-X, Sanchez-Alonso P, Schreier P-H, HäuserHahn I, Vaupel M, Koopmann E, Friedrich G, Voss H, Schlüter T, Margolis J, Platt D, Swimmer C, Gnirke A, Chen F, Vysotskaia V, Mannhaupt G, Güldener U, Münsterkötter M, Haase D, Oesterheld M, Mewes H-W, Mauceli E-W, DeCaprio D, 
564

565

566

567

568

569

570

571

572

573

574

575

576

577

578

579

580

581

582

583

584

585

586

587

588

589

590

591

592

Wade C-M, Butler J, Young S, Jaffe D-B, Calvo S, Nusbaum C, Galagan J, Birren B-W. 2006. Insights from the genome of the biotrophic fungal plant pathogen Ustilago maydis. Nature 444: 97-101

Kuratani M, Tanaka K, Terashima K, Muraguchi H, Nakazawa T, Nakahori K, Kamada T. 2010. The dst 2 gene essential for photomorphogenesis of Coprinopsis cinerea encodes a protein with a putative FAD-binding-4 domain. Fungal Genetics and Biology 47(2): 152-158

Langfelder P, Horvath S. 2008. WGCNA: an R package for weighted correlation network analysis. BMC bioinformatics 9(1): 559-571

Lee J-Y, Kim L-H, Kim H-E, Park J-S, Han K-H, Han D-M. 2013. A putative APSES transcription factor is necessary for normal growth and development of Aspergillus nidulans. The Journal of Microbiology. 51(6): 800-806

Lee M-K, Kwon N-J, Choi J-M, Lee I-S, Jung S, Yu J-H. 2014. NsdD is a key repressor of asexual development in Aspergillus nidulans. Genetics 197(1): 159-173

Lentjes M-H, Niessen H-E, Akiyama Y, de Bruïne A-P, Melotte V, van Engeland M. 2016. The emerging role of GATA transcription factors in development and disease. Expert Reviews in Molecular Medicine, 18: e3.

Lescot M, Déhais P, Thijs G, Marchal K, Moreau Y, Yves V-D-P, Pieree R, Stephane R. 2002. Plantcare, a database of plant cis-acting regulatory elements and a portal to tools for in silico analysis of promoter sequences. Nucleic Acids Research 30(1): 325-327

Letunic I, Doerks T, Bork P. 2012. SMART 7: recent updates to the protein domain annotation resource. Nucleic Acids Research 40(1): 302-305

Li J, Mu W, Veluchamy S, Liu, Zhang Y,Pan H, Rollins J-A. 2018. The GATA-type IVb zincfinger transcription factor SsNsd1 regulates asexual-sexual development and appressoria formation in Sclerotinia sclerotiorum. Molecular Plant Pathology 19: 1679-1689

Lin Q-Y, Li T-H, Huang H-H, Song B. 2009. Studies on light and temperature conditions for cultivation of Cordyceps guangdongensis. Journal of South China Agricultural University 30 (1): $42-45$

Linden H, Macino G. 1997. White collar 2, a partner in blue-light signal transduction, controlling expression of light-regulated genes in Neurospora crassa. EMBO J 16: 98-109 
593 Liu L, Wang Q, Sun Y, Zhang Y, Zhang X, Liu J, Gao Y, Pan H, 2018. Sssfh1, a gene encoding

594

595

596

597

598

599

600

601

602

603

604

605

606

607

608

609

610

611

612

613

614

615

616

617

618

619

620

621

622

623

a putative component of the RSC chromatin remodeling complex, is involved in hyphal growth, reactive oxygen species accumulation, and pathogenicity in Sclerotinia sclerotiorum. Frontiers in microbiology 9: 1828

Liu L, Wang Q, Zhang X, Liu J, Zhang Y, Pan H. 2018. Ssams2, a gene encoding GATA transcription factor, is required for appressoria formation and chromosome segregation in Sclerotinia sclerotiorum. Frontiers in microbiology 9: 3031

Livak K-J, Schmittgen T-D. 2001. Analysis of relative gene expression data using real-time quantitative PCR and the $2^{-\Delta \Delta \mathrm{Ct}}$ method. Methods 25(4): 402-408

Lowry J-A, Atchley W-R. 2000. Molecular evolution of the GATA family of transcription factors: conservation within the DNA-binding domain. Journal of. Molecular Evolution 50(2): 103-115

Machida M, Gomi K. 2010. Aspergillus: Molecular Biology and Genomics. Wymondham: Horizon Scientific Press.

McGinnis S, Madden T-L. 2004. BLAST: at the core of a powerful and diverse set of sequence analysis tools. Nucleic Acids Research 32(suppl_2): 20-25

Michielse C, Pfannmüller A, Macios M, Rengers P, Dzikowska A, Tudzynski B. 2014. The interplay between the GATA transcription factors AreA, the global nitrogen regulator and AreB in Fusarium fujikuroi. Molecular microbiology 91: 472-493

Mihlan M, Homann V, Liu T-D, Tudzynski B. 2003. AreA directly mediates nitrogen regulation of gibberellin biosynthesis in Gibberella fujikuroi, but its activity is not affected by NMR. Molecular microbiology 47(4): 975-991

Niehaus E-M, Schumacher J, Burkhardt I, Rabe P, Spitzer E, Münsterkötter M, Güldener U, Sieber C-M-K, Dickschat J-S, Tudzynski B. 2017. The GATA-type transcription factor Csm1 regulates conidiation and secondary metabolism in Fusarium fujikuroi. Frontiers in microbiology 8: 1175-1191

Nierman W-C, Pain A, Anderson M-J, Wortman J-R, Kim H-S, Arroyo J, Berriman M, Abe K, Archer D-B, Bermejo C, Bennett J, Bowyer P, Chen D, Collins M, Coulsen R, Davies R, Dyer P-S, Farman M, Fedorova N, Fedorova N, Feldblyum T-V, Fischer R, Fosker N, Fraser A, García J-L, García M-J, Goble A, Goldman G-H, Gomi K, Griffith-Jones S, Gwilliam R, Haas B, Haas H, Harris D, Horiuchi H, Huang J, Humphray S, Jiménez J, 
624

625

626

627

628

629

630

631

632

633

634

635

636

637

638

639

640

641

642

643

644

645

646

647

648

649

650

651

652

Keller N, Khouri H, Kitamoto K, Kobayashi T, Konzack S, Kulkarni R, Kumagai T, Lafton A, Latgé J-P, Li W-X, Lord A, Lu C, Majoros W-H, May G-S, Miller B-L, Mohamoud Y, Molina M, Monod M, Mouyna I, Mulligan S, Murphy L, O'Neil S, Paulsen I, Peñalva M-A, Pertea M, Price C, Pritchard B-L, Quail M-A, Rabbinowitsch E, Rawlins N, Rajandream M-A, Reichard U, Renauld H, Robson G-D, de Córdoba SR, Rodríguez-Peña J-M, Ronning C-M, Rutter S, Salzberg S-L, Sanchez M, SánchezFerrero J-C, Saunders D, Seeger K, Squares R, Squares S, Takeuchi M, Tekaia F, Turner G, de Aldana C-R-V, Weidman J, White O, Woodward J, Yu J-H, Fraser C, Galagan J-E, Asai K, Machida M, Hall N, Barrell B, Denning D-W. 2005. Genomic sequence of the pathogenic and allergenic filamentous fungus Aspergillus fumigatus. Nature 438: 1151-1156

Oberegger H, Schoeser M, Zadra I, Abt B, Haas H. 2001. SREA is involved in regulation of siderophore biosynthesis, utilization and uptake in Aspergillus nidulans. Molecular microbiology 41(5): 1077-1089

Park J, Kim H, Kim S, Kong S-H, Park J, Kim S, Han H-Y, Park B-S, Jung K-Y, Lee Y-H. 2006. A comparative genome-wide analysis of GATA transcription factors in Fungi. Genomics Inform. 4: 147-160

Patient R-K, McGhee J-D. 2002. The GATA family (vertebrates and invertebrates). Current Opinion in Genetics and Development 12(4): 416-422

Pfannmüller A, Leufken J, Studt L, Michielse C-B, Sieber C-M-K, Güldener U, Hawat S, Hippler M, Fufezan C, Tudzynski B. 2017. Comparative transcriptome and proteome analysis reveals a global impact of the nitrogen regulators AreA and AreB on secondary metabolism in Fusarium fujikuroi. PLoS One 12: e0176194

Quispe C-F. 2011. GATA-family transcription factors in Magnaporthe oryzae. Teses, Dissertations, and Student Research in Agronomy and Horticulture 30.

Reyes J-C, Muro-Pastor M-I, Florencio F-J. 2004. The GATA family of transcription factors in arabidopsis and rice. Plant Physiology 134(4): 1718-1732

Rodriguez-Romero J, Maren H, Christian K, Sylvia M, Reinhard F. 2010. Fungi, hidden in soil or up in the air: light makes a difference. Annual Review of Microbiology 64: 585-610 
653

654

655

656

657

658

659

660

661

662

663

664

665

666

667

668

669

670

671

672

673

674

675

676

677

678

679

680

681

682

683

Ronsmans A, Wery M, Szachnowski U, Gautier C, Descrimes M, Dubois E, Morillon A, Georis I. 2019. Transcription-dependent spreading of the Dal80 yeast GATA factor across the body of highly expressed genes. PLoS Genetics 15(2): e1007999.

Sano H, Kaneko S, Sakamoto Y, Sato T. 2009. The basidiomycetous mushroom Lentinula edodes white collar-2 homolog PHRB, a partner of putative blue-light photoreceptor PHRA, binds to a specific site in the promoter region of the L. edodes tyrosinase gene. Fungal Genetics and Biology 46(4): 333-341

Sanz C, Rodríguez-Romero J. Idnurm A. Christie J-M. 2009. Phycomyces MADB interacts with MADA to form the primary photoreceptor complex for fungal phototropism. Proceedings of the National Academy of Sciences of the United States of America 106(17): 7095-7100

Scazzocchio C. 2000. The fungal GATA factors. Current Opinion in Microbiology 3(2): 126-131

Schumacher J. 2017. How light a $\square$ ects the life of Botrytis. Fungal Genetics and Biology 106: $26-41$

Schumacher J. 2012. Tools for Botrytis cinerea: New expression vectors make the gray mold fungus more accessible to cell biology approaches. Fungal Genetics and Biology 49(6): 483497

Schumacher J, Simon A, Cohrs K-C, Viaud M, Tudzynski P. 2014. The transcription factor BcLTF1 regulates virulence and light responses in the necrotrophic plant pathogen Botrytis cinerea. PLoS Genetics 10(1): e1004040

Shannon P, Markiel A, Ozier O, Baliga N-S, Wang J-T, Ramage D, Amin N, Schwikowski B, Ideker T. 2003. Cytoscape: A Software Environment for Integrated Models of Biomolecular Interaction Networks. Genome Research 13(11): 2498-2504

Sil A, Herskowitz I. 1996. Identification of asymmetrically localized determinant, Ashlp, required for lineage-specific transcription of the yeast HO gene. Cell 84(5): 711-722

Tamura K, Peterson D, Peterson N, Stecher G, Nei M, Kumar S. 2011. MEGA5: molecular evolutionary genetics analysis using maximum likelihood, evolutionary distance, and maximum parsimony methods. Molecular Biology and Evolution 28(10): 2731-2739

Thompson J-D, Gibson,T-J, Plewniak F, Jeanmougin F, Higgins D-G. 1997. The Clustal X windows interface: flexible strategies for multiple sequence alignment aided by quality analysis tools. Nucleic Acids Research 25: 4876-4882

Peer) reviewing PDF | (2020:01:44976:2:1:NEW 21 Jul 2020) 
684 Tremblay M, Sanchez-Ferras O, Bouchard M. 2018. GATA transcription factors in 685 development and disease. Development 145: dev164384

686 Wang G-Z, Cheng H-J, Li M, Zhang C-H, Deng W-Q, Li T-H. 2020. Selection and validation 687 of reliable reference genes for Tolypocladium guangdongense gene expression analysis under

688

689

690

691

692

693

694

695

696

697

698

699

700

701

702

703

704

705

706

707

708

709

710

711

712 differentially developmental stages and temperature stresses. Gene 734: 144380

Wong K-H, Hynes M-J, Davis M-A. 2008. Recent advances in nitrogen regulation: a comparison between Saccharomyces cerevisiae and filamentous fungi. Eukaryotic Cell 7(6): 917-925

Yang T, Dong C. 2014. Photo morphogenesis and photo response of the bluelight receptor gene Cmwc-1 in different strains of Cordyceps militaris. FEMS Microbiology Letters 352(2): 190197

Yang T, Guo M, Yang H, Guo S, Dong C. 2016. The blue-light receptor $C m W C$ - 1 mediates fruit body development and secondary metabolism in Cordyceps militaris. Applied Microbiology and Biotechnology 100(2): 743-755

Yang T, Xiong W, Dong C. 2013. Cloning and analysis of the $O s w c-1$ gene encoding a putative blue light photoreceptor from Ophiocordyceps sinensis. Mycoscience 55(4): 241-245

Yu Z-Z, Fischer R. 2018. Light sensing and responses in fungi. Nature Reviews Microbiology 112

Yu M, Yu J-J, Cao H-J, Yong M-L, Liu Y-F. 2019. Genome-wide identification and analysis of the GATA transcription factor gene family in Ustilaginoidea virens. Genome 62: 807-816

Zdobnov E-M, Apweiler R. 2001. InterProScan-an integration platform for the signaturerecognition methods in InterPro. Bioinformatics 17(9): 847-848

Zhang B, Horvath S. 2005. A general framework for weighted gene co-expression network analysis. Statistical Applications in Genetics and Molecular Biology 4(1): Article 17

Zhang C-H, Huang H, Deng W-Q, Li T-H. 2019. Genome-wide analysis of the Zn(II)2Cys6 zinc cluster-encoding gene family in Tolypocladium guangdongense and its light-induced expression. Genes 10(3): 179-198

Zhang C, Deng W, Yan W, Li T. 2018. Whole genome sequence of an edible and potential medicinal fungus, Cordyceps guangdongensis. G3-Genes Genomes. Genetics. 8(6): 18631870 
713

714

715

716

717

718

719

720

721

722

723

724

725

726

727

728

729

730 731 Ascomycota.

732

Table S6 List of the identified motif and their characteristics identified by MEME. X, Huang W. 2015. Genome-wide survey of the soybean GATA transcription factor gene family and expression analysis under low nitrogen stress. PLoS One 10: e0125174

Zhang Z, Ren C, Zou L, Wang Y, Li S-H, Liang Z-C. 2018. Characterization of the GATA gene family in Vitis vinifera: genome-wide analysis, expression profiles, and involvement in light and phytohormone response. Genome 61: 713-723

Zhang Z, Zou X, Huang Z, Fan S, Qun G, Liu A, Gong J, Li J, Gong W, Shi Y, Fan L, Zhang Z, Liu R, Jiang X, Lei K, Shang H, Xu A, Yuan Y. 2019. Genome-wide identification and analysis of the evolution and expression patterns of the GATA transcription factors in three species of Gossypium genus. Gene 680: 72-83

Table 1 Characterization of GATA-TFs in Tolypocladium guangdongense.

Table 2. Comparison of GATA-TFs between Tolypocladium guangdongense and other fungi.

Table S1 Primers used in this study for qPCR analyses.

Table S2 Amino acid sequences and chromosome positions of TgGATAs.

Table S3 Detail information of homologous proteins of TgGATAs.

Table S4 Intron-exon structures in $\operatorname{Tg} G A T A s$.

Table S5 The numbers of amino acid and introns position in TgGATAs and their homologs in

Zhang C, Hou Y, Hao Q, Chen H, Chen L, Yuan S, Shan Z, Zhang X, Yang Z, Qiu D, Zhou

Table S7 List of the predicted cis-elements and their functions. 
734 Table S8 Stability analysis of eight candidate reference genes for qPCR analysis in Tolypocladium

735 guangdongensis under different light conditions calculated by the GeNorm and NormFinder

736 analysis, respectively.

737 Table S9 Pairwise Pearson correlation coefficients of TgGATAs analyzed by WGCNA according

738 to the previous RNA-seq data.

739 Figure legends

740 Figure 1. GATA-domain analysis of the identified GATA-TFs in Tolypocladium guangdongense.

741 The threshold for consensus highlighting was 30\%. The black asterisk denoted consensus

742 sequences, and red star represents the seventh position of the $\mathrm{Zn}$ finger loop.

743 Figure 2. Phylogenetic tree of GATA-TFs from Tolypocladium guangdongense and other fungi

744 in three classes of Ascomycota, including Eurotiomycetes, Leotiomycetes and Sordariomycetes.

745 The phylogenetic tree was constructed using the NJ (neighbor-joining) method with 1000

746 bootstrap replications. The different classes are distinguished by different colors. Detailed

747 information on the homologous protein is provided in Supplementary Table 3.

748 Figure 3. Intron-exon structure analysis of GATA-TFs in Tolypocladium guangdongense.

749 Information regarding the intron and exon positions are provided in Supplementary Table 4.

750 Figure 4. Intron/exon structures of TgGATA1-5 and their homologous genes in other fungi. Intron

751 positions in the GATA-TFs of Tolypocladium guangdongense and other fungi are denoted by

752 different colored triangles on the amino acid sequences. Sequences 1 to 13 represent the

753 corresponding orthologs in Tolypocladium guangdongense, Tolypocladium ophioglossoides,

754 Tolypocladium paradoxum, Ophiocordyceps sinensis, Fusarium fujikuroi, Fusarium oxysporum,

755 Cordyceps militaris, Neurospora crassa, Magnaporthe oryzae, Botrytis cinerea, Sclerotinia 
756 sclerotiorum, Aspergillus nidulans, Aspergillus niger. Detailed information on the number of

757 amino acids and the intron positions are provided in Supplementary Table 5.

758 Figure 5. Motif analysis of GATA-TFs in Tolypocladium guangdongense and known functional

759 GATA-TFs in other fungi. The phylogenetic tree was constructed using the NJ (neighbor-joining)

760 method with 1000 bootstrap replications. Ten conserved motifs were identified in the proteins and

761 are indicated in different colored boxes. $\mathrm{ZnF}$, represented the GATA-type Zinc finger domain.

762 PAS, represented the PAS domain. UN, represented uncharacterized motif. The scale bar indicated

763 the number of amino acids (aa). Each motif sequence and alignment is shown in Supplementary

764 Table 6 and Supplementary Figure 1. An, A. nidulans; Bc, B. cinerea; Cm, C. militaris; Ff, F.

765 fujikuroi; Nc, N. crassa; Os, O. sinensis; Ss, S. sclerotiorum; Tg, T. guangdongense. GenBank

766 numbers of known functional GATA-TFs are listed as follows: AnNsdD, AAB16914; AnSREA,

767 AAD25328; Bcltf1, ANQ80444; BcWC-1, XP_024547291; CmWC-1, EGX96523; Ffcsm1,

768 CCT68588; NcADS4, AAG45180; NcNIT2, P19212; NcSREA, EAA32742; NcSub-1,

769 ESA42507; NcWC-1， Q01371; NcWC-2， EAA34583; OsWC-1， EQK98623; Ssams2,

770 SS1G_03252; Sssfh1, SS1G_01151; SsNsd1, ANQ80447.

771 Figure 6. Structural features and light-responsive cis-element analysis of GATA-TFs in

772 Tolypocladium guangdongense. A, Schematic representation of GATA-TFs in T. guangdongense.

773 The positions of the LOV domain, the PAS domain and the zinc-finger domain were predicted

774 using the SMART program (http://smart.embl-heiderberg.de/). B, Analyses of cis-elements in

775 promoter regions of $\operatorname{TgGATAs}$. The promoter sequences (-1500 bp) of TgGATAs were used for

776 analyses. The different types of cis-elements are indicated by various geometric figures at the

777 corresponding positions, and detailed information is listed in Supplementary Table 7. 
778 Figure 7. Quantitative real-time PCR analyses of TgGATAs in response to light. Gene expression 779 was measured after different illumination times. The mean expression value was calculated from 780 three independent replicates. The vertical bars indicate the standard deviation. Expression level 781 was normalized by the selected reference genes $\alpha$-tub-1 (A) and $\beta$-tub-l(B).

782 Figure 8. Quantitative real-time PCR analyses of $\operatorname{Tg} G A T A s$ during fruiting body development. 783 Gene expression was measured in different developmental stages, including vegetative stage (M), 784 color transition period (TC), primordia (P), early stage of fruiting body development (FB1), middle 785 stage of fruiting body development (FB2), late stage of fruiting body development (FB3), maturing 786 period of fruiting body (FM). The mean expression value was calculated from three independent 787 replicates. The vertical bars indicate the standard deviation. Expression level was normalized by 788 the selected reference genes VPS (A) and $H 4(\mathrm{~B})$.

789 Figure S1 Motif sequences in TgGATAs and other known functional GATA-TFs.

790 Figure S2 Amino acid alignments of the Zn finger loops in TgGATA1-TgGATA5 and their 791 homologous proteins.

792 
Table $\mathbf{1}$ (on next page)

Characterization of GATA-TFs in Tolypocladium guangdongense. 
1 Table 1 Characterization of GATA-TFs in Tolypocladium guangdongense.

\begin{tabular}{cccccccc}
\hline Gene name & Gene ID & Location & aa & Exon & PI & Mw(Da) & Subcellular location \\
\hline TgGATA1 & CCG_01872 & Chromosome 1 & 1034 & 2 & 7.3 & 112095.44 & Nucleus* \\
TgGATA2 & CCG_04254 & Chromosome 2 & 541 & 3 & 5.51 & 59186.27 & Nucleus \\
TgGATA3 & CCG_07987 & Chromosome 4 & 979 & 3 & 9.24 & 102733.69 & Nucleus* \\
TgGATA4 & CCG_02574 & Chromosome 1 & 376 & 4 & 5.54 & 40488.91 & Nucleus \\
TgGATA5 & CCG_07433 & Chromosome 4 & 535 & 3 & 9.07 & 56338.17 & Nucleus \\
TgGATA6 & CCG_06499 & Chromosome 3 & 195 & 3 & 11.08 & 20884.24 & Nucleus* \\
TgGATA7 & CCG_00651 & Chromosome 1 & 462 & 1 & 7.22 & 50682.75 & Mitochondrion \\
\hline
\end{tabular}

2 * represented nuclear localization signal sequences found in amino acid sequences. Detailed information of the TgGATAs are provided in Table S2. 
Table 2 (on next page)

Comparison of GATA-TFs between Tolypocladium guangdongense and other fungi. 
1 Table 2 Comparison of GATA-TFs between Tolypocladium guangdongense and other fungi.

\begin{tabular}{|c|c|c|c|c|c|c|c|c|c|c|c|}
\hline \multirow{3}{*}{$\begin{array}{l}\square \\
\text { Ascomycota } \\
\text { Dothideomycetes }\end{array}$} & \multirow{3}{*}{$\begin{array}{l}\text { Family } \\
\square \\
\square\end{array}$} & \multirow{3}{*}{$\begin{array}{l}\text { Species } \\
\square \\
\square\end{array}$} & \multirow{3}{*}{$\begin{array}{l}\square \\
1 \\
\square\end{array}$} & \multirow{3}{*}{$\begin{array}{c}\square \\
2 \\
\square\end{array}$} & \multirow{3}{*}{$\begin{array}{c}\square \\
\mathbf{3} \\
\square\end{array}$} & \multicolumn{2}{|c|}{ GATAs $^{\mathrm{a}}$} & \multirow{3}{*}{$\begin{array}{l}\square \\
6 \\
\square\end{array}$} & \multirow{3}{*}{$\begin{array}{l}\square \\
7 \\
\square\end{array}$} & \multicolumn{2}{|c|}{ Other GATAs ${ }^{b}$} \\
\hline & & & & & & 4 & 5 & & & Number & \multirow[t]{2}{*}{ Reference $^{c}$} \\
\hline & & & & & & $\square$ & $\square$ & & & $\square$ & \\
\hline Pleosporales & Leptosphaeriaceae & Leptosphaeria maculans & $\sqrt{ }$ & $\sqrt{ }$ & $\sqrt{ }$ & l & $\sqrt{ }$ & / & / & l & - \\
\hline Capnodiales & Mycosphaerellaceae & Zymoseptoria tritici & $\sqrt{ }$ & $\sqrt{ }$ & $\sqrt{ }$ & $\sqrt{ }$ & $\sqrt{ }$ & / & / & l & - \\
\hline Eurotiomycetes & $\square$ & $\square$ & $\square$ & $\square$ & $\square$ & $\square$ & $\square$ & $\square$ & $\square$ & $\square$ & $\square$ \\
\hline Eurotiales & Aspergillaceae & Aspergillus fumigatus & $\sqrt{ }$ & $\sqrt{ }$ & $\sqrt{ }$ & $\sqrt{ }$ & $\sqrt{ }$ & $\sqrt{ }$ & $\sqrt{ }$ & 1 & Nierman et al., 2005 \\
\hline Eurotiales & Aspergillaceae & Aspergillus flavus & $\sqrt{ }$ & $\sqrt{ }$ & $\sqrt{ }$ & $\sqrt{ }$ & $\sqrt{ }$ & $\sqrt{ }$ & $\sqrt{ }$ & l & - \\
\hline Eurotiales & Aspergillaceae & Aspergillus nidulans & $\sqrt{ }$ & $\sqrt{ }$ & $\sqrt{ }$ & $\sqrt{ }$ & $\sqrt{ }$ & $\sqrt{ }$ & / & 1 & Lowry and Atchley, 2000 \\
\hline Eurotiales & Aspergillaceae & Aspergillus niger & $\sqrt{ }$ & $\sqrt{ }$ & $\sqrt{ }$ & $\sqrt{ }$ & $\sqrt{ }$ & $\sqrt{ }$ & / & l & Park et al., 2006 \\
\hline Eurotiales & Aspergillaceae & Aspergillus terreus & $\sqrt{ }$ & $\sqrt{ }$ & $\sqrt{ }$ & $\sqrt{ }$ & $\sqrt{ }$ & / & 1 & / & FTFD \\
\hline Eurotiales & Aspergillaceae & Penicillium chrysogenum & $\sqrt{ }$ & $\sqrt{ }$ & $\sqrt{ }$ & $\sqrt{ }$ & $\sqrt{ }$ & / & / & 5 & FTFD \\
\hline Eurotiales & Aspergillaceae & Penicillium marneffei & $\sqrt{ }$ & $\sqrt{ }$ & $\sqrt{ }$ & $\sqrt{ }$ & $\sqrt{ }$ & $\sqrt{ }$ & ' & 5 & FTFD \\
\hline Eurotiales & Aspergillaceae & Talaromyces stipitatus & $\sqrt{ }$ & $\sqrt{ }$ & $\sqrt{ }$ & $\sqrt{ }$ & $\sqrt{ }$ & / & l & 6 & FTFD \\
\hline Onygenales & Ajellomycetaceae & Histoplasma capsulatum & $\sqrt{ }$ & l & $\sqrt{ }$ & $\sqrt{ }$ & / & / & l & 2 & Park et al., 2006 \\
\hline Leotiomycetes & $\square$ & $\square$ & $\square$ & $\square$ & $\square$ & $\square$ & $\square$ & $\square$ & $\square$ & $\square$ & $\square$ \\
\hline Helotiales & Drepanopezizaceae & Marssonina brunnea & $\sqrt{ }$ & $\sqrt{ }$ & $\sqrt{ }$ & $\sqrt{ }$ & $\sqrt{ }$ & / & l & 1 & - \\
\hline Helotiales & Helotiaceae & Glarea lozoyensis & $\sqrt{ }$ & $\sqrt{ }$ & $\sqrt{ }$ & $\sqrt{ }$ & $\sqrt{ }$ & / & / & l & - \\
\hline Helotiales & Ploettnerulaceae & Rhynchosporium commune & $\sqrt{ }$ & $\sqrt{ }$ & $\sqrt{ }$ & $\sqrt{ }$ & $\sqrt{ }$ & / & l & 1 & - \\
\hline Thelebolales & Thelebolaceae & Pseudogymnoascus destructans & $\sqrt{ }$ & $\sqrt{ }$ & $\sqrt{ }$ & $\sqrt{ }$ & $\sqrt{ }$ & / & l & 1 & - \\
\hline Helotiales & Sclerotiniaceae & Botrytis cinerea & $\sqrt{ }$ & $\sqrt{ }$ & $\sqrt{ }$ & $\sqrt{ }$ & $\sqrt{ }$ & $\sqrt{ }$ & & 1 & Park et al., 2006 \\
\hline Helotiales & Sclerotiniaceae & Sclerotinia sclerotiorum & $\sqrt{ }$ & $\sqrt{ }$ & $\sqrt{ }$ & $\sqrt{ }$ & $\sqrt{ }$ & $\sqrt{ }$ & / & 2 & Park et al., 2006; Li et al., 2018; Liu et al., 2018 \\
\hline Pezizomycetes & $\square$ & $\square$ & $\square$ & $\square$ & $\square$ & $\square$ & $\square$ & $\square$ & $\square$ & $\square$ & $\square$ \\
\hline Pezizales & Tuberaceae & Tuber borchii & $\sqrt{ }$ & $\sqrt{ }$ & $\sqrt{ }$ & $\sqrt{ }$ & $\sqrt{ }$ & / & / & l & Ambra et al., 2004 \\
\hline Pezizales & Tuberaceae & Tuber melanosporum & $\sqrt{ }$ & $\sqrt{ }$ & $\sqrt{ }$ & $\sqrt{ }$ & $\sqrt{ }$ & / & / & / & - \\
\hline Saccharomycetes & $\square$ & $\square$ & $\square$ & $\square$ & $\square$ & $\square$ & $\square$ & $\square$ & $\square$ & $\square$ & $\square$ \\
\hline Saccharomycetales & Dipodascaceae & Yarrowia lipolytica & $\sqrt{ }$ & l & $\sqrt{ }$ & $\sqrt{ }$ & $\sqrt{ }$ & / & l & 5 & Dujon et al., 2004 \\
\hline Saccharomycetales & Saccharomycetaceae & Saccharomyces cerevisiae & $\mathrm{N}$ & $\mathrm{N}$ & / & $\sqrt{ }$ & / & / & l & 10 & Lowry and Atchley, 2000; Park et al., 2006 \\
\hline
\end{tabular}




\begin{tabular}{|c|c|c|c|c|c|c|c|c|c|c|c|}
\hline Sordariomycetes & $\square$ & $\square$ & $\square$ & $\square$ & $\square$ & $\square$ & $\square$ & $\square$ & $\square$ & $\square$ & $\square$ \\
\hline Glomerellales & Glomerellaceae & Colletotrichum graminicola & $\sqrt{ }$ & $\sqrt{ }$ & $\sqrt{ }$ & $\sqrt{ }$ & $\sqrt{ }$ & l & l & l & - \\
\hline Hypocreales & Cordycipitaceae & Cordyceps militaris & $\sqrt{ }$ & $\sqrt{ }$ & $\sqrt{ }$ & $\sqrt{ }$ & $\sqrt{ }$ & / & l & / & - \\
\hline Hypocreales & Hypocreaceae & Trichoderma atroviride & $\sqrt{ }$ & $\sqrt{ }$ & $\sqrt{ }$ & $\sqrt{ }$ & $\sqrt{ }$ & l & l & 2 & FTFD \\
\hline Hypocreales & Hypocreaceae & Trichoderma reesei & $\sqrt{ }$ & $\sqrt{ }$ & $\sqrt{ }$ & $\sqrt{ }$ & $\sqrt{ }$ & 1 & l & 2 & Park et al., 2006 \\
\hline Hypocreales & Incertae sedis & Acremonium chrysogenum & $\sqrt{ }$ & $\sqrt{ }$ & $\sqrt{ }$ & $\sqrt{ }$ & $\sqrt{ }$ & / & & / & - \\
\hline Hypocreales & Clavicipitaceae & Ustilaginoidea virens & $\sqrt{ }$ & $\sqrt{ }$ & $\sqrt{ }$ & $\sqrt{ }$ & $\sqrt{ }$ & I & l & 2 & Yu et al., 2019 \\
\hline Hypocreales & Nectriaceae & Fusarium fujikuroi & $\sqrt{ }$ & $\sqrt{ }$ & $\sqrt{ }$ & $\sqrt{ }$ & $\sqrt{ }$ & $\sqrt{ }$ & $\sqrt{ }$ & l & Michielse et al., 2014; Niehaus et al., 2017 \\
\hline Hypocreales & Nectriaceae & Fusarium oxysporum & $\sqrt{ }$ & $\sqrt{ }$ & $\sqrt{ }$ & $\sqrt{ }$ & $\sqrt{ }$ & / & l & 3 & FTFD \\
\hline Hypocreales & Ophiocordycipitaceae & Ophiocordyceps sinensis & $\sqrt{ }$ & $\sqrt{ }$ & $\sqrt{ }$ & & $\sqrt{ }$ & 1 & / & / & - \\
\hline Hypocreales & Ophiocordycipitaceae & Tolypocladium guangdongense & $\sqrt{ }$ & $\sqrt{ }$ & $\sqrt{ }$ & $\sqrt{ }$ & $\sqrt{ }$ & $\sqrt{ }$ & $\sqrt{ }$ & / & this study \\
\hline Hypocreales & Ophiocordycipitaceae & Tolypocladium ophioglossoides & $\sqrt{ }$ & $\sqrt{ }$ & & $\sqrt{ }$ & $\sqrt{ }$ & / & l & l & - \\
\hline Hypocreales & Ophiocordycipitaceae & Tolypocladium paradoxum & $\sqrt{ }$ & $\sqrt{ }$ & $\sqrt{ }$ & $\sqrt{ }$ & $\sqrt{ }$ & $\sqrt{ }$ & $\sqrt{ }$ & l & - \\
\hline Magnaporthales & Magnaporthaceae & Magnaporthe oryzae & $\sqrt{ }$ & $\sqrt{ }$ & $\sqrt{ }$ & $\sqrt{ }$ & $\sqrt{ }$ & 1 & / & 4 & Dean et al., 2005; FTFD \\
\hline Sordariales & Chaetomiaceae & Chaetomium globosum & $\sqrt{ }$ & $\sqrt{ }$ & $\sqrt{ }$ & $\sqrt{ }$ & 1 & / & / & 1 & Park et al., 2006; FTFD \\
\hline Sordariales & Sordariaceae & Neurospora crassa & $\sqrt{ }$ & $\sqrt{ }$ & $\sqrt{ }$ & $\sqrt{ }$ & $\sqrt{ }$ & $\sqrt{ }$ & / & 1 & Borkovich et al., 2004, Li et al., 2018 \\
\hline Basidiomycota & $\square$ & $\square$ & $\square$ & $\square$ & $\square$ & $\square$ & $\square$ & $\square$ & $\square$ & $\square$ & $\square$ \\
\hline Agaricomycetes & $\square$ & $\square$ & $\square$ & $\square$ & $\square$ & $\square$ & $\square$ & $\square$ & $\square$ & $\square$ & $\square$ \\
\hline Agaricales & Psathyrellaceae & Coprinopsis cinerea & $\sqrt{ }$ & 1 & / & $\sqrt{ }$ & $\sqrt{ }$ & $\sqrt{ }$ & l & / & - \\
\hline Agaricales & Pleurotaceae & Pleurotus ostreatus & $\sqrt{ }$ & 1 & ' & $\sqrt{ }$ & 1 & 1 & $\sqrt{ }$ & 6 & FTFD \\
\hline Tremellomycetes & $\square$ & $\square$ & $\square$ & $\square$ & $\square$ & $\square$ & $\square$ & $\square$ & $\square$ & $\square$ & $\square$ \\
\hline Tremellales & Tremellaceae & Cryptococcus neoformans & $\sqrt{ }$ & $\sqrt{ }$ & $\sqrt{ }$ & $\sqrt{ }$ & $\sqrt{ }$ & / & l & 7 & Park et al., 2006 \\
\hline Ustilaginomycetes & $\square$ & $\square$ & $\square$ & $\square$ & $\square$ & $\square$ & $\square$ & $\square$ & $\square$ & $\square$ & $\square$ \\
\hline Ustilaginales & Ustilaginaceae & Ustilago maydis & $\sqrt{ }$ & $\sqrt{ }$ & $\sqrt{ }$ & & $\sqrt{ }$ & l & $\sqrt{ }$ & 5 & Kamper et al., 2006 \\
\hline
\end{tabular}

2 a GATA1-7 represent the corresponding homologues of TgGATA1-7. /, represents that non- homologue or lower homology GATA transcription factor was found.

3 ' Other GATAs represent the non- homologues or lower homology GATAs with TgGATA1-7 in each species.

$4 \quad{ }^{\mathrm{c}}$-, the homologues of TgGATA1-7 were identified by genome-wide Blast analyses; FTFD, the total number of GATAs was obtained from the Fungal Transcription Factor Database (FTFD); Some of

5 GATAs and the number of GATAs in special species have been reported by previous studies. 


\section{Figure 1}

GATA-domain analysis of the identified GATA-TFs in Tolypocladium guangdongense.

The threshold for consensus highlighting was 30\%. The black asterisk denoted consensus sequences, and red star represents the seventh position of the Zn finger loop.

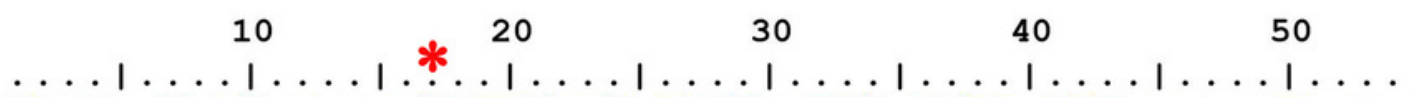

TgGATA1 GNMVRDCANCHTRNTPEWRRGPSGQRDLCNSCGLRWAKQTGRVSPRNSSRGGN- (901-953)

TgGATA2 TSEEYVCTDCGTLDSPEWRKGPSGPKTLCNACGLRWAKKEKKRN--NTSQPMVD (486-537)

TgGATA6 GNARLECDACGTSETPEWRC GPD GLGTLCNVCGLVLAKKORR-------RSGV- (150-195)

TgGATA7 SYQQFICRHCGTSDTSLWRKDTHD-RLLCNACGH-YLQRIGGVRAIFAINAPS- (13-63)

TgGATA4 GGNLPTCQNCTTSTTPLWRRDEFG-SVLCNACGL-FLKLHGRPRPISLKTDVI- (70-120)

TgGATA5-1 SQGGOVCSNCGTTRTPLWRRSPQG-ATICNACGL-YLKARNTSRPTSLKKPPH- (71-121) TgGATA5-2 NTVVIACQNCGTTITPLWKRDESG-HTICNACGL-YYKLHGVHRPVTMKKATI- (228-278) TgGATA3 GSQPTTCTNCFTQTTPLWRRNPEG-QPLCNACGL-FLKLHGVVRPLSLKTDVI- (704-754) 


\section{Figure 2}

Phylogenetic tree of GATA-TFs from Tolypocladium guangdongense and other fungi in three classes of Ascomycota, including Eurotiomycetes, Leotiomycetes and Sordariomycetes.

The phylogenetic tree was constructed using the NJ (neighbor-joining) method with 1000 bootstrap replications. The different classes are distinguished by different colors. Detailed information on the homologous protein is provided in Supplementary Table 3. 


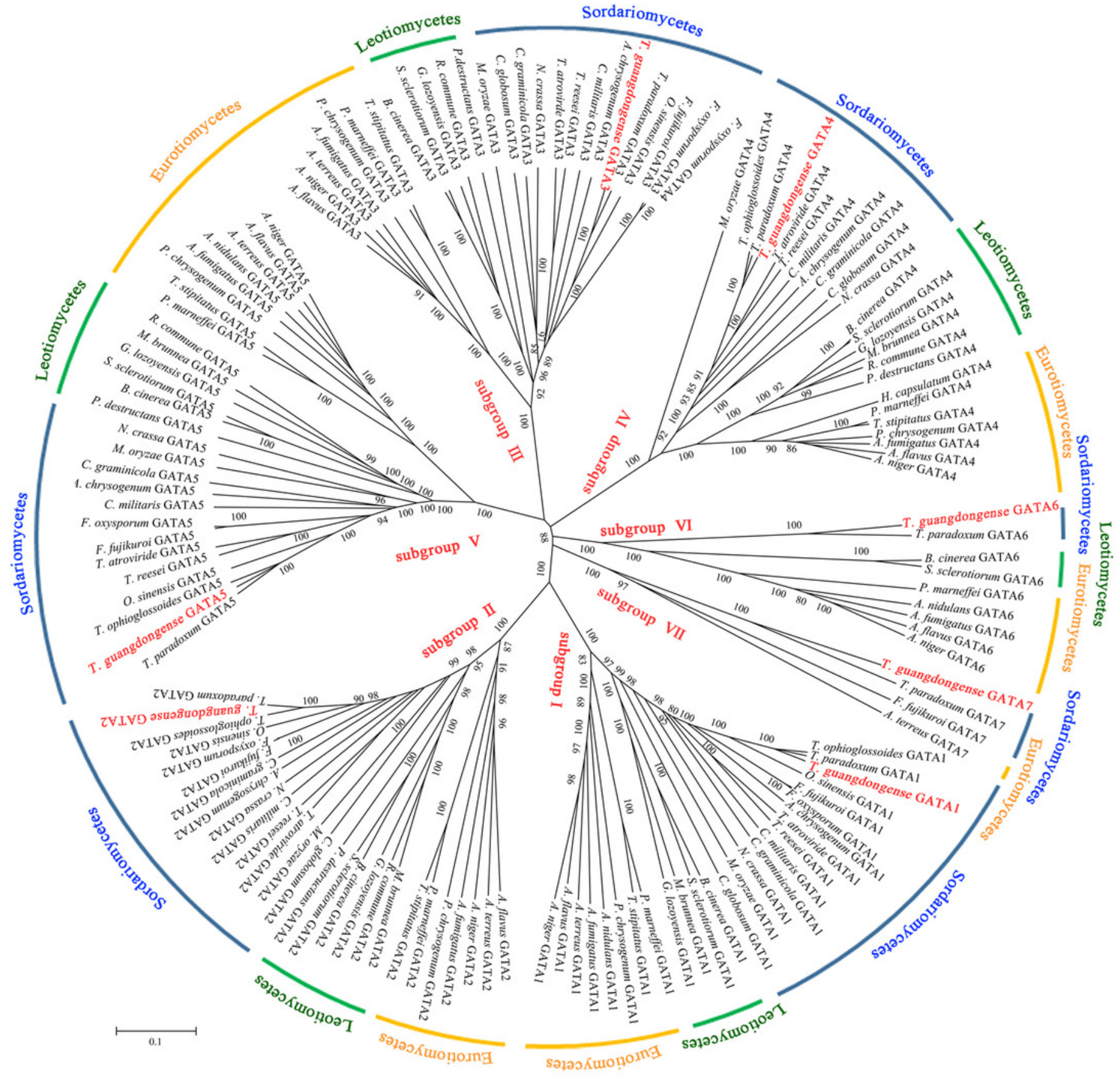


Figure 3

Intron-exon structure analysis of GATA-TFs in Tolypocladium guangdongense.

Information regarding the intron and exon positions are provided in Supplementary Table 4.

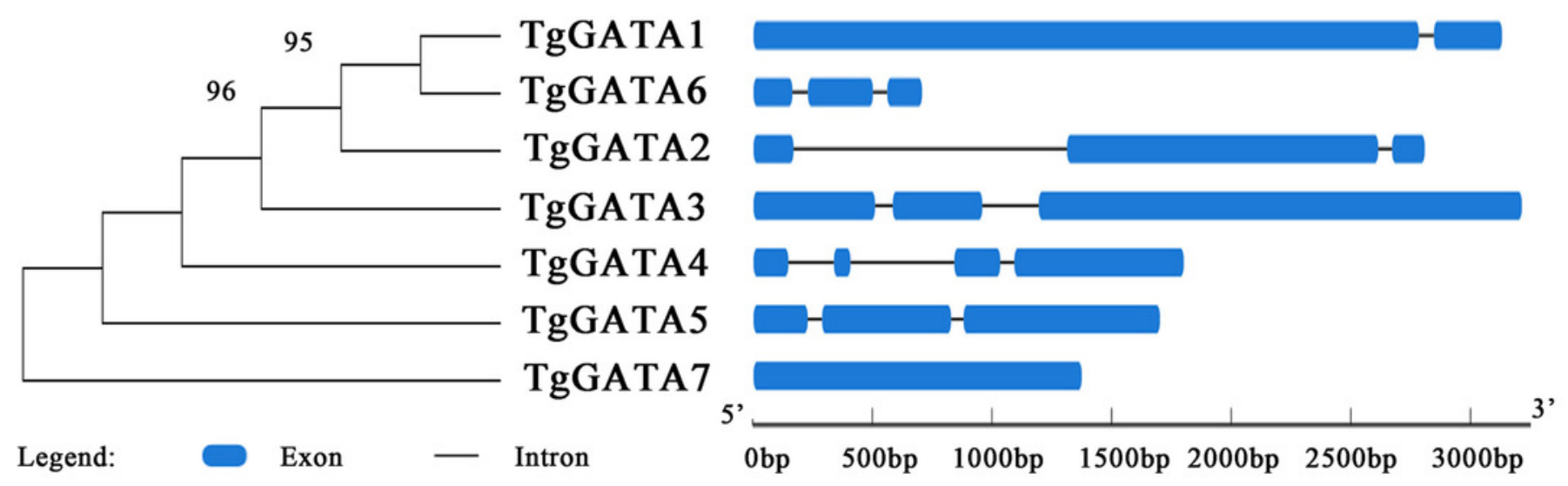




\section{Figure 4}

Intron/exon structures of TgGATA1-5 and their homologous genes in other fungi.

Intron positions in the GATA-TFs of Tolypocladium guangdongense and other fungi are denoted by different colored triangles on the amino acid sequences. Sequences 1 to 13 represent the corresponding orthologs in Tolypocladium guangdongense, Tolypocladium ophioglossoides, Tolypocladium paradoxum, Ophiocordyceps sinensis, Fusarium fujikuroi, Fusarium oxysporum, Cordyceps militaris, Neurospora crassa, Magnaporthe oryzae, Botrytis cinerea, Sclerotinia sclerotiorum, Aspergillus nidulans, Aspergillus niger. Detailed information on the number of amino acids and the intron positions are provided in Supplementary Table 5. 

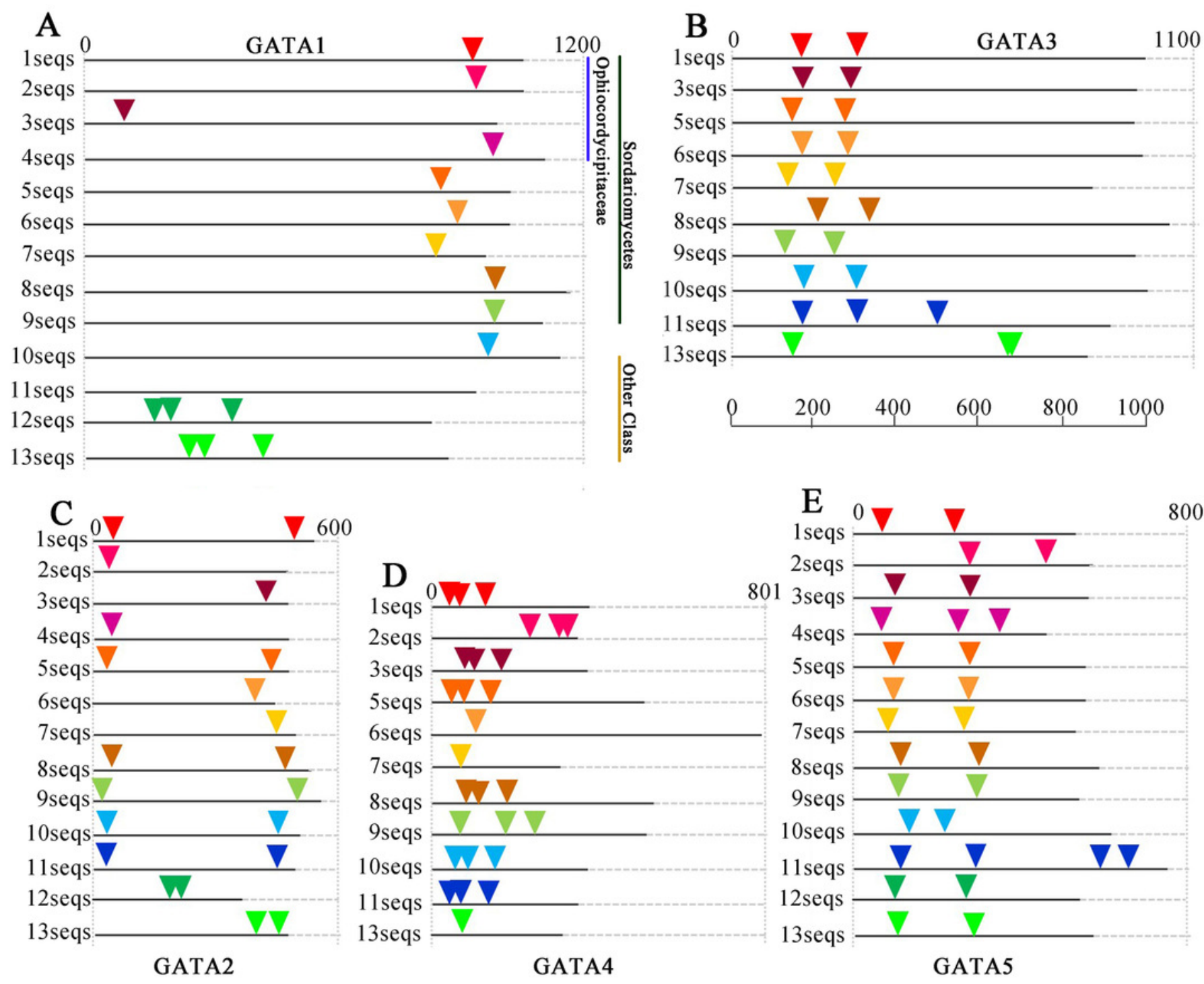


\section{Figure 5}

Motif analysis of GATA-TFs in Tolypocladium guangdongense and known functional GATA-TFs in other fungi.

The phylogenetic tree was constructed using the NJ (neighbor-joining) method with 1000 bootstrap replications. Ten conserved motifs were identified in the proteins and are indicated in different colored boxes. ZnF, represented the GATA-type Zinc finger domain. PAS, represented the PAS domain. UN, represented uncharacterized motif. The scale bar indicated the number of amino acids (aa). Each motif sequence and alignment is shown in Supplementary Table 6 and Supplementary Figure S1. An, A. nidulans; Bc, B. cinerea; Cm, C. militaris; Ff, F. fujikuroi; Nc, N. crassa; Os, O. sinensis; Ss, S. sclerotiorum; Tg, T. guangdongense. GenBank numbers of known functional GATA-TFs are listed as follows: AnNsdD, AAB16914; AnSREA, AAD25328; Bcltf1, ANQ80444; BcWC-1, XP_024547291; CmWC-1, EGX96523; Ffcsm1, CCT68588; NcADS4, AAG45180; NCNIT2, P19212; NcSREA, EAA32742; NcSub-1, ESA42507; NcWC-1, Q01371; NcWC-2, EAA34583; OsWC-1, EQK98623; Ssams2, SS1G_03252; Sssfh1, SS1G_01151; SsNsd1, ANQ80447. 


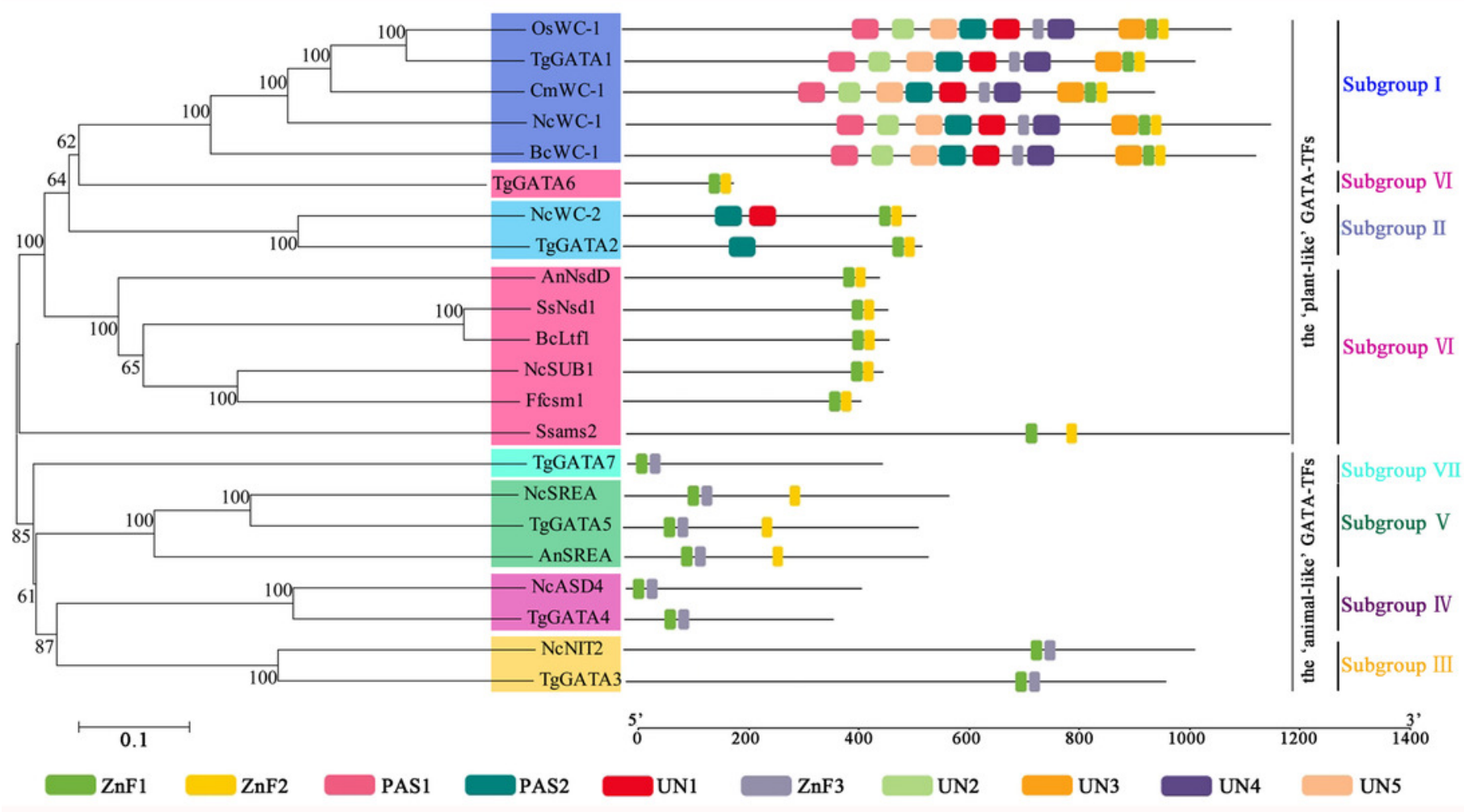




\section{Figure 6}

Structural features and light-responsive cis-element analysis of GATA-TFs in Tolypocladium guangdongense.

A, Schematic representation of GATA-TFs in T. guangdongense. The positions of the LOV domain, the PAS domain and the zinc-finger domain were predicted using the SMART program ( http://smart.embl-heiderberg.de/ ). B, Analyses of cis-elements in promoter regions of TgGATAs. The promoter sequences (-1500 bp) of TgGATAs were used for analyses. The different types of cis-elements are indicated by various geometric figures at the corresponding positions, and detailed information is listed in Supplementary Table 7.

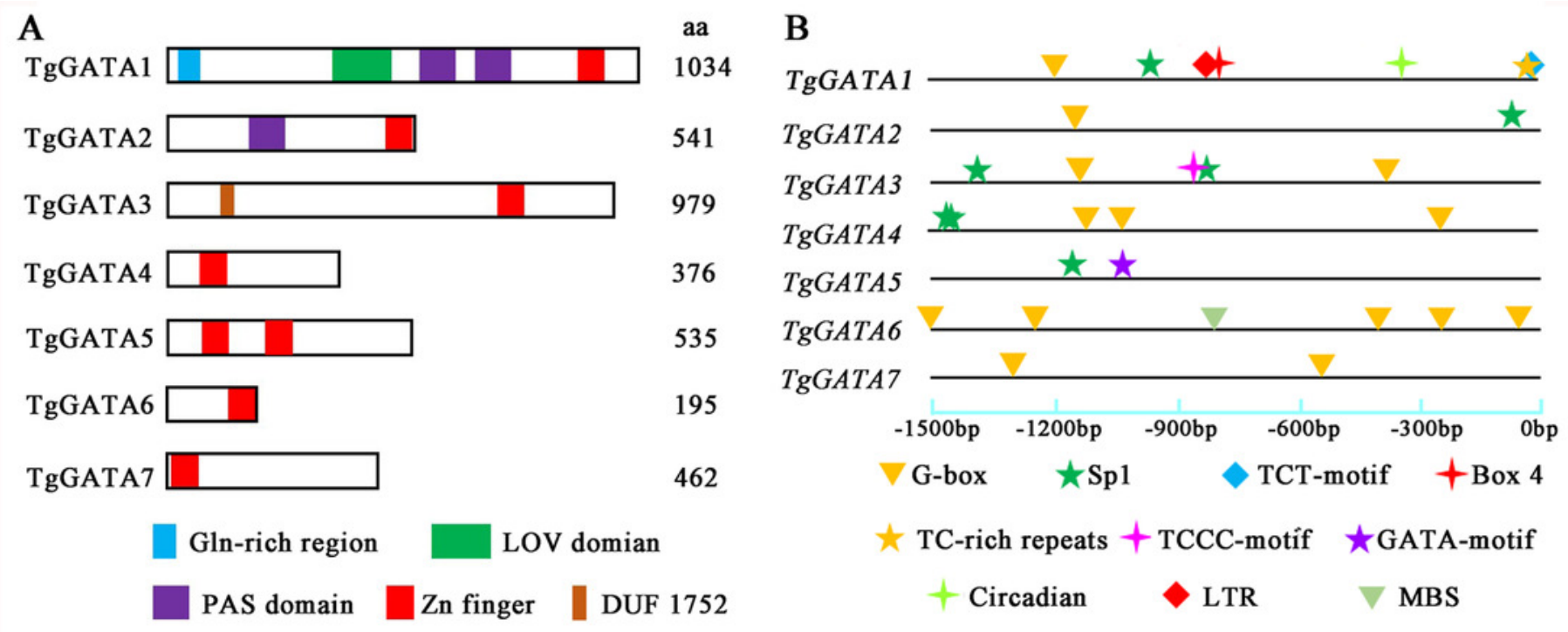




\title{
Figure 7
}

Quantitative real-time PCR analyses of TgGATAs in response to light.

Gene expression was measured after different illumination times. The mean expression value was calculated from three independent replicates. The vertical bars indicate the standard deviation. Expression level was normalized by the selected reference genes $\alpha$-tub-1 (A) and $\beta$-tub-1(B).

\section{A 3.5}

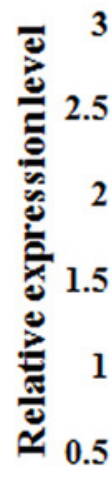

0
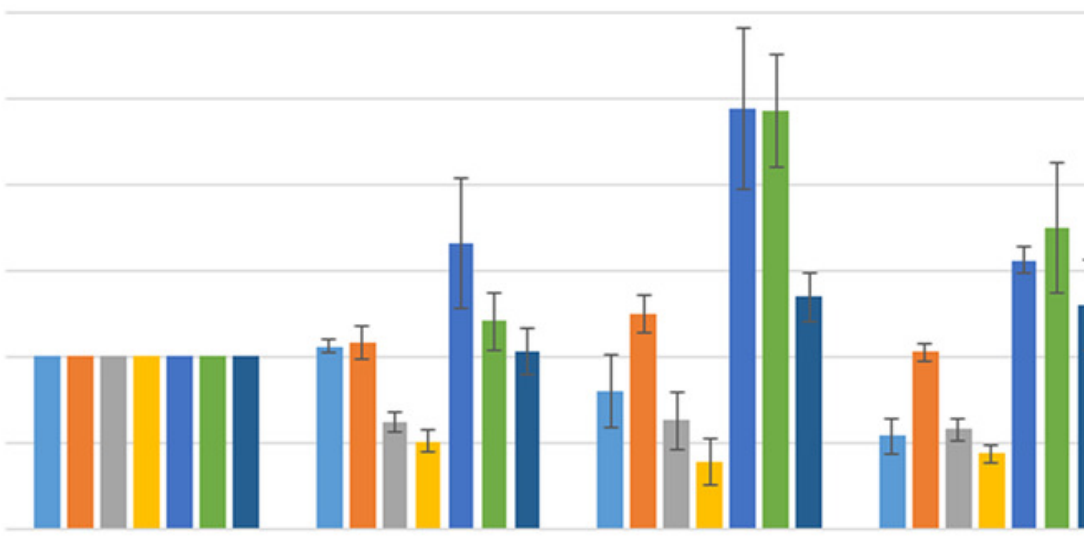

$\because T g G A T A 1$

$\because T g G A T A 2$

$\because T g G A T A 3$

- TgGATA4

- TgGATAS

- TgGATA6

- TgGATA7

\begin{abstract}
Dark
\end{abstract}
L-15min

L-30min

L-2h

L-4h

B 3

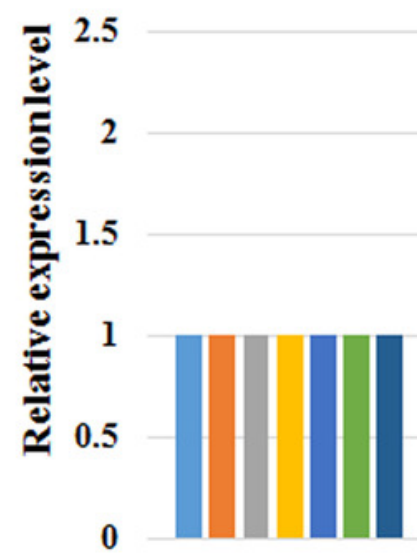

Dark

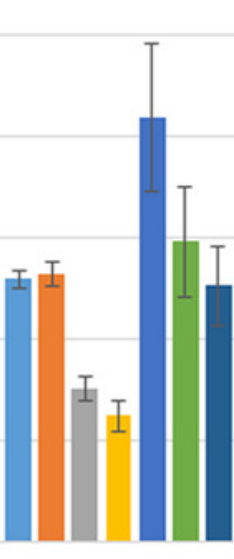

L-15min

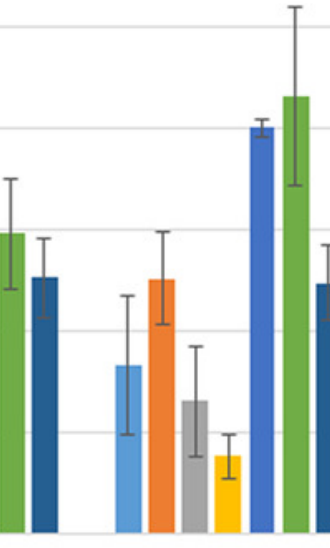

L-30min

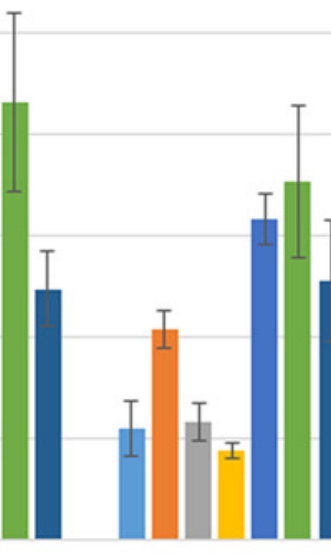

L-2h $\square T g G A T A 1$

$\backsim T g G A T A 2$

$\square T g G A T A 3$

$\because \operatorname{TgGATA4}$

- TgGATA5

๓ TgGATA6

-TgGATA7 


\section{Figure 8}

Quantitative real-time PCR analyses of TgGATAs during fruiting body development.

Gene expression was measured in different developmental stages, including vegetative stage (M), color transition period (TC), primordia (P), early stage of fruiting body development (FB1), middle stage of fruiting body development (FB2), late stage of fruiting body development (FB3), maturing period of fruiting body (FM). The mean expression value was calculated from three independent replicates. The vertical bars indicate the standard deviation. Expression level was normalized by the selected reference genes VPS (A) and $H 4$ (B). 

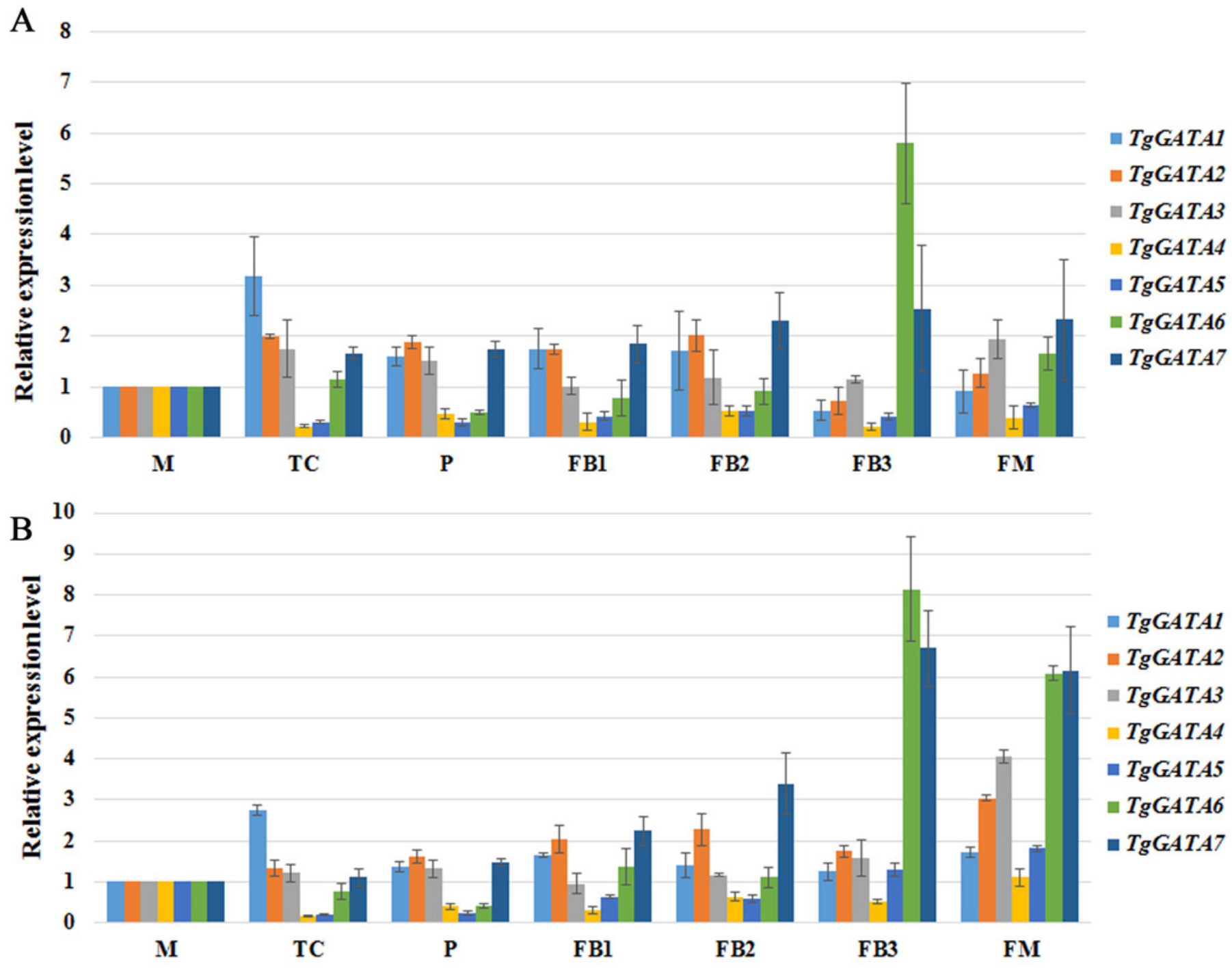